\title{
Non-fragile multivariable PID controller design via system augmentation ${ }^{\dagger}$
}

\author{
Jinrong Liu*, James Lam, Mouquan Shen and Zhan Shu \\ Department of Mechanical Engineering, The University of Hong Kong, Pokfulam Road, Hong Kong
}

(Received 00 Month 20XX; final version received 00 Month 20XX)

\begin{abstract}
In this paper, the issue of designing non-fragile $H_{\infty}$ multivariable PID controllers with derivative filters is investigated. In order to obtain the controller gains, the original system is associated with an extended system such that the PID controller design can be formulated as a static output-feedback (SOF) control problem. By taking the system augmentation approach, the conditions with slack matrices for solving the non-fragile $H_{\infty}$ multivariable PID controller gains are established. Based on the results, linear matrix inequality (LMI) based iterative algorithms are provided to compute the controller gains. Simulations are conducted to verify the effectiveness of the proposed approaches.
\end{abstract}

Keywords: $H_{\infty}$ control; linear matrix inequality (LMI); non-fragile control; PID control

\section{Introduction}

Proportional-integral-derivative (PID) controllers are found in almost all areas of control application. It is estimated that more than 90\% control loops are of PID type and actually most of them are with the derivative gain set to zero (PI control)(Knospe 2006). Due to its popularity in industrial applications, many formulations of PID controller gain tuning have been proposed since the past decades (Åström and Hägglund 2006), and even in recent years. For instance, a methodology for tuning the gains of fuzzy PID controllers is proposed by taking explicitly into account the closed-loop system performance(Gil et al. 2015). In Jung et al. (2015), an adaptive PID speed control scheme for permanent magnet synchronous motor drives is developed. For open-flow canal control systems, a gain-scheduled Smith Predictor PIDbased LPV controller is proposed in Bolea et al. (2014). A neural PID control approach is proposed for robot manipulators with application to an upper limb exoskeleton (Yu and Rosen 2013). Some novel tuning approaches of fractional order PID controllers for control systems are proposed recently(Fergani and Charef 2016; Bettou and Charef 2009).

For industrial processes of multivariable nature, sometimes multivariable control approaches must be used to achieve satisfactory performances. This motivates to derive effective approaches to designing PID controllers for multi-inputs multi-outputs (MIMO) systems (Gündeş et al. 2009; Zhang et al. 2012; Wu et al. 2011; Vu and Lee 2010; Bianchi et al. 2008; Zhang et al. 2004). Decentralised PID controllers which are also referred to as multi-loop PID controllers prevail in multivariable processes because they have the advantage of a simpler structure and, accordingly, require fewer parameters to tune (Palmor et al. 1995). However, in some situations a decentralised PID controller may fail to achieve a satisfactory performance when loop interaction is significant. Though introducing some decoupling techniques (Wang et al. 1997; Halevi et al. 1997; Wu et al. 2009; Jin and Liu 2014) may improve these situations, it may still fail especially for high-dimensional systems of which loop interactions are severe. In such cases, a centralised PID controller is desirable. As compared with a PID controller of decentralised type, the difficulty for tuning a centralised PID controller lies in much more gain parameters should be tuned because the whole loop inter-

\footnotetext{
†This work was partially supported by GRF HKU 201411159139.

*Corresponding author. Email: liujinrjason@gmail.com
} 
action of a system has been considered. In the past decades, a lot of effort has been devoted to this area, and some research works have been proposed to tune a centralised PID controller via LMI approaches (Zheng et al. 2002; Lin et al. 2004; He and Wang 2006). For these approaches it is often assumed that PID controllers are always implemented exactly. It is worth pointing out that in the implementation of controllers, many factors should be considered, for example, finite word length in digital microprocessors, the imprecision inherent in analog systems and the expectation of allowable tuning for the implemented controller gains (Keel and Bhattacharyya 1997). This fact leads to the so-called non-fragile or resilient control problem. Actually, a lot of research works related to such a problem have been investigated in the past decades (Yang and Wang 2001; Yang and Che 2008; Zhang et al. 2014; Che et al. 2014; Shen et al. 2014; Yue and Lam 2005; Tandon and Dhawan 2016; Huang and Yang 2015). Norm-bounded gain perturbations are considered in the control for active vehicle suspensions (Du et al. 2003) and uncertain linear neutral delay systems (Xu et al. 2004). In Shu et al. (2009), a special expression is used to model the multiplicative controller gain variations in the stabilization for a class of discrete-time linear systems with missing data in actuators. Recently, the stochastic perturbations are also considered in the filtering and control for networked control systems (Hu et al. 2015; Li et al. 2015). Moreover, it is noted that many previous research works of nonfragile control are concerned with the state-feedback control, output-feedback control and filtering. These motivate us to design PID controllers which are insensitive to gain perturbations, that is, non-fragile PID controllers. Although, there are a few results concerning non-fragile PID control (Ho 2000; Ho et al. 2001; Li et al. 2006), only single-input single-output (SISO) systems are considered. Another practical problem that arises in industrial PID control is the noise effect of the derivative action. Most of the previous results concerning PID controller designs often do not work properly since they often ignore this effect. It is known that differentiation is always sensitive to noise and thus the derivative action of practical PID controllers may amplify high-frequency noise. Then the input signal can be so large that the performance of the controlled system may become poor and the system may become unstable in the end. Such an issue can be solved by introducing a low-pass filter which helps to limit the high-frequency gain (Åström and Hägglund 2006). A PID controller with a derivative filter is often referred to as a PIDF controller and some results on the PIDF controllers for SISO systems can be found in (Knospe 2006; Hägglund 2012, 2013). However, to the authors'knowledge, little results about PIDF control are available for MIMO systems, especially for the case with controller gain perturbations considered.

With the above background as motivation, we study in this paper the issue of non-fragile PID controller design for MIMO systems. In particular, we focus on the issue of synthesizing a stabilizing PID controller for a linear continuous-time system while satisfying an $H_{\infty}$ norm performance with a prescribed level. To tackle this issue, we extend the original system to an associated system such that the PID control problem can be formulated into designing a static output-feedback (SOF) controller. Also, additive controller gain perturbations and multiplicative controller gain perturbations are considered in the controller design. Following the idea of system augmentation (Shu and Lam 2009), the conditions with slack matrices for solving non-fragile $H_{\infty}$ MIMO PID controller gains are established. Based on these results, a suitable PID control law can be constructed in terms of a solution to a certain matrix inequality. Linear matrix inequality (LMI) based iterative algorithms are provided to compute the controller gains. Finally, simulations are conducted to verify the effectiveness of the non-fragile design.

Notations: Throughout this paper, for real symmetric matrices $X$ and $Y$, the notation $X \geq Y$ (respectively, $X>Y$ ) means that the matrix $X-Y$ is positive semi-definite (respectively, positive definite). $I$ is the identity matrix with appropriate dimension. The superscript $\mathrm{T}$ represents the transpose. For a transfer function matrix $G(s),\|G\|_{\infty}$ represents the $H_{\infty}$ norm of $G(s)$. The symbol $*$ is used to denote a matrix which can be inferred by symmetry. The shorthand notion $\operatorname{Sym}(X)$ represents $X+X^{\mathrm{T}}$. Matrices are assumed to have compatible dimensions for algebraic operations if not explicitly stated. 


\section{Preliminaries}

Consider the following linear continuous-time system:

$$
\left\{\begin{array}{l}
\dot{x}(t)=A x(t)+B u(t)+B_{w} w(t) \\
z(t)=C x(t)+D_{z u} u(t)+D_{z w} w(t) \\
y(t)=C_{y} x(t)
\end{array}\right.
$$

and a PID controller with a derivative (first-order low-pass) filter:

$$
\left\{\begin{array}{l}
u(t)=K_{\mathrm{P}} y(t)+K_{\mathrm{I}} \int_{0}^{t} y(\eta) \mathrm{d} \eta+K_{\mathrm{D}} y_{\mathrm{D}}(t) \\
\mathscr{T}_{\dot{y}_{\mathrm{D}}}(t)+y_{\mathrm{D}}(t)=\dot{y}(t)
\end{array}\right.
$$

where $x(t) \in \mathbb{R}^{n}$ is the state vector, $u(t) \in \mathbb{R}^{r}$ is the input vector, $y(t) \in \mathbb{R}^{m}$ is the output vector, $A, B, B_{w}, C$, $D_{z u}, D_{z w}$ and $C_{y}$ are system matrices with appropriate dimensions. Matrix $\mathscr{T}:=\operatorname{diag}\left(\tau_{1}, \tau_{2}, \ldots, \tau_{r}\right)$ and $\tau_{j}>$ 0 for $j=1,2, \ldots, r$, are the time constants. $K_{\mathrm{P}}, K_{\mathrm{I}}, K_{\mathrm{D}} \in \mathbb{R}^{r \times m}$ are gains of the PID controller parameters to be determined. For notational convenience, let $K=\left[K_{\mathrm{P}} K_{\mathrm{I}} K_{\mathrm{D}}\right]$.

Remark 1. The filter in the PID controllers design provides the advantages of removing the undesired noise components from the measurement signal and compensating for the undesired dynamics in the process (Hägglund 2012). Proper filtering action is of great significance for the overall performance of the control system, especially when the controller is a PID one, since the structure of this controller has limitations(Romero Segovia et al. 2013; Hägglund 2013). In this paper, the first-order low-pass filter is introduced to the derivative term of the controller as shown in (2).

The objective of the PID controller design is to find a set of gains $K_{\mathrm{P}}, K_{\mathrm{I}}, K_{\mathrm{D}}$ such that the control system fulfills given specifications or performances. Note that inaccuracies and uncertainties occurring in the implementation of a designed controller do exist. It is necessary to consider the gain perturbations of the PID controller parameters, which can be described as $\Delta K_{\mathrm{P}}, \Delta K_{\mathrm{I}}, \Delta K_{\mathrm{D}}$ with respect to $K_{\mathrm{P}}, K_{\mathrm{I}}, K_{\mathrm{D}}$. Let $\Delta K=\left[\Delta K_{\mathrm{P}} \Delta K_{\mathrm{I}} \Delta K_{\mathrm{D}}\right]$. In this paper, two types of controller gain perturbations will be considered as follows.

1) Norm-bounded additive perturbations:

$$
\Delta K_{\mathrm{P}}=\mathscr{M}_{1} F_{1} \mathscr{N}_{1}, \Delta K_{\mathrm{I}}=\mathscr{M}_{2} F_{2} \mathscr{N}_{2}, \Delta K_{\mathrm{D}}=\mathscr{M}_{3} F_{3} \mathscr{N}_{3}
$$

2) Norm-bounded multiplicative perturbations:

$$
\Delta K_{\mathrm{P}}=K_{\mathrm{P}} \tilde{\mathscr{M}}_{1} F_{1} \mathscr{N}_{1}, \Delta K_{\mathrm{I}}=K_{\mathrm{I}} \tilde{\mathscr{M}}_{2} F_{2} \mathscr{N}_{2}, \Delta K_{\mathrm{D}}=K_{\mathrm{D}} \tilde{\mathscr{M}}_{3} F_{3} \mathscr{N}_{3}
$$

where $\mathscr{M}_{i}, \tilde{\mathscr{M}}_{i}$ and $\mathscr{N}_{i}$ for $i=1,2,3$, are known matrices with appropriate dimensions and $F_{i}$ for $i=1,2,3$, are unknown constant matrices satisfying

$$
F_{i}^{\mathrm{T}} F_{i} \leq I
$$

Remark 2. The multiplicative perturbation form described in (4) represents controller input perturbation. The dual case: $\Delta K_{\mathrm{P}}=\tilde{\mathscr{M}}_{1} F_{1} \mathscr{N}_{1} K_{\mathrm{P}}, \Delta K_{\mathrm{I}}=\tilde{\mathscr{M}}_{2} F_{2} \mathscr{N}_{2} K_{\mathrm{I}}, \Delta K_{\mathrm{D}}=\tilde{\mathscr{M}}_{3} F_{3} \mathscr{N}_{3} K_{\mathrm{D}}$, which corresponds to controller output perturbation, will not be considered in the exposition. However, it can be treated similarly using the system augmentation approach.

The issue of designing non-fragile $H_{\infty}$ PID controllers (NFHPID) in this paper is addressed as follows. 
Problem NFHPID: Design a PID controller:

$$
\left\{\begin{array}{l}
u(t)=\left(K_{\mathrm{P}}+\Delta K_{\mathrm{P}}\right) y(t)+\left(K_{\mathrm{I}}+\Delta K_{\mathrm{I}}\right) \int_{0}^{t} y(\eta) \mathrm{d} \eta+\left(K_{\mathrm{D}}+\Delta K_{\mathrm{D}}\right) y_{\mathrm{D}}(t) \\
\mathscr{T} \dot{y}_{\mathrm{D}}(t)+y_{\mathrm{D}}(t)=\dot{y}(t)
\end{array}\right.
$$

such that the linear continuous-time system in (1) is asymptotically stable and satisfied:

$$
\left\|T_{z w}\right\|_{\infty}<\gamma, \quad \gamma>0
$$

where $\Delta K_{\mathrm{P}}, \Delta K_{\mathrm{I}}, \Delta K_{\mathrm{D}}$ are the controller gain perturbations defined in (3)-(5), $T_{z w}$ represents the operator of linear continuous-time system in (1) from $w(t)$ to $z(t)$.

In order to find $K$, we can extend the linear continuous-time system with a PID controller to another system by defining a new state vector $\bar{x}(t)=\left[x(t)^{\mathrm{T}} \int_{0}^{t} y(\eta)^{\mathrm{T}} \mathrm{d} \eta \mathscr{T} y_{\mathrm{D}}(t)^{\mathrm{T}}\right]^{\mathrm{T}}$ and a new output vector $\bar{y}(t)=$ $\left[y(t)^{\mathrm{T}} \int_{0}^{t} y(\eta)^{\mathrm{T}} \mathrm{d} \eta y_{\mathrm{D}}(t)^{\mathrm{T}}\right]^{\mathrm{T}}$, respectively. Thus an extended system is obtained as follows:

$$
\left\{\begin{array}{l}
\dot{\bar{x}}(t)=\bar{A} \bar{x}(t)+\bar{B} u(t)+\bar{B}_{w} w(t) \\
z(t)=\bar{C} \bar{x}(t)+D_{z u} u(t)+D_{z w} w(t) \\
\bar{y}(t)=\bar{C}_{y} \bar{x}(t)
\end{array}\right.
$$

with an equivalent PID controller of SOF form:

$$
u(t)=(K+\Delta K) \bar{y}(t)
$$

where

$$
\begin{gathered}
\bar{A}=\left[\begin{array}{ccc}
A & 0 & 0 \\
C_{y} & 0 & 0 \\
C_{y} A & 0 & -\mathscr{T}^{-1}
\end{array}\right], \quad \bar{B}=\left[\begin{array}{c}
B \\
0 \\
C_{y} B
\end{array}\right] \\
\bar{B}_{w}=\left[\begin{array}{c}
B_{w} \\
0 \\
C_{y} B_{w}
\end{array}\right], \quad \bar{C}=\left[\begin{array}{lll}
C & 0 & 0
\end{array}\right], \quad \bar{C}_{y}=\left[\begin{array}{ccc}
C_{y} & 0 & 0 \\
0 & I & 0 \\
0 & 0 & \mathscr{T}^{-1}
\end{array}\right]
\end{gathered}
$$

This SOF control system in (8)-(9) can be rewritten in closed-loop form:

$$
\left\{\begin{array}{l}
\dot{\bar{x}}(t)=\hat{A} \bar{x}(t)+\bar{B}_{w} w(t) \\
z(t)=\hat{C} \bar{x}(t)+D_{z w} w(t)
\end{array}\right.
$$

where $\hat{A}=\bar{A}+\bar{B}(K+\Delta K) \bar{C}_{y}$ and $\hat{C}=\bar{C}+D_{z u}(K+\Delta K) \bar{C}_{y}$. Through this extension, the issue of non-fragile $H_{\infty}$ PID controller design is reduced to the following problem.

Problem SOF: Design a SOF controller:

$$
u(t)=(K+\Delta K) \bar{y}(t)
$$

such that the closed-loop system in (10) is asymptotically stable and satisfied

$$
\left\|\bar{T}_{z w}\right\|_{\infty}<\gamma, \quad \gamma>0
$$


where $\Delta K$ is the controller gain variation, $\bar{T}_{z w}$ represents the operator of linear continuous-time system in (10) from $w(t)$ to $z(t)$. Note that $\bar{T}_{z w}=T_{z w}$, and thereby in order to solve Problem NFHPID, it is sufficient to solve Problem SOF.

Remark 3. Through the aforementioned manipulation, Problem NFHID is formulated as Problem SOF. The advantage of this formulation lies in the fact that no assumptions or conditions are needed while structural assumption and condition are considered in Zheng et al. (2002); Lin et al. (2004); He and Wang (2006). For example, in Zheng et al. (2002), $C_{y} B_{w}=0$ and $I-K_{\mathrm{D}} C_{y} B$ invertible are needed for solving the $H_{\infty}$ control problem.

Note that $K+\Delta K$ is embedded between $\bar{B}$ and $\bar{C}_{y}$, and $D_{z u}$ and $\bar{C}_{y}$. By the system augmentation approach (Shu and Lam 2009), a state vector $\tilde{x}(t)=\left[\bar{x}(t)^{\mathrm{T}} u(t)^{\mathrm{T}}\right]^{\mathrm{T}}$ is introduced, and the closed-loop system in (10) can be further augmented to give

$$
\left\{\begin{array}{l}
\mathbf{E} \dot{\tilde{x}}(t)=\mathbf{A} \tilde{x}(t)+\mathbf{B}_{w} w(t) \\
z(t)=\mathbf{C} \tilde{x}(t)+\mathbf{D} w(t)
\end{array}\right.
$$

where

$$
\mathbf{E}=\left[\begin{array}{ll}
I & 0 \\
0 & 0
\end{array}\right], \quad \mathbf{A}=\left[\begin{array}{cc}
\bar{A} & \bar{B} \\
(K+\Delta K) \bar{C}_{y} & -I
\end{array}\right], \quad \mathbf{B}_{w}=\left[\begin{array}{c}
\bar{B}_{w} \\
0
\end{array}\right], \quad \mathbf{C}=\left[\begin{array}{ll}
\bar{C} & D_{z u}
\end{array}\right], \quad \mathbf{D}=D_{z w}
$$

In this augmentation representation, $K+\Delta K$ has been separated from $\bar{B}$ and $\bar{C}_{y}$, and $D_{z u}$ and $\bar{C}_{y}$, which makes matrix parameterizing more flexible. Before ending this section, the following proposition and lemma which are useful in the sequel are given.

Proposition 1. For $\Delta K \equiv 0$, the following statements are equivalent:

1) The linear continuous-time system in (10) is asymptotically stable and satisfied $\left\|\bar{T}_{z w}\right\|_{\infty}<\gamma, \gamma>0$.

2) There exists a matrix $P_{1}>0$ such that

$$
\left[\begin{array}{ccc}
\operatorname{Sym}\left(\hat{A}^{\mathrm{T}} P_{1}\right) & P_{1} \bar{B}_{w} & \hat{C}^{\mathrm{T}} \\
* & -\gamma I & D_{z w}^{\mathrm{T}} \\
* & * & -\gamma I
\end{array}\right]<0
$$

3) There exist matrices $P_{1}>0, P_{2}>0$ and a sufficiently large scalar $\alpha>0$ such that

$$
\Theta=\left[\begin{array}{cr}
\operatorname{Sym}\left(\mathscr{A}^{\mathrm{T}} \mathscr{P}\right)+\Lambda & \mathscr{S}^{\mathrm{T}} \\
* & -\gamma I
\end{array}\right]<0
$$

where

$$
\begin{array}{cc}
\mathscr{A}=\left[\begin{array}{ccc}
\bar{A} & \bar{B} & \bar{B}_{w} \\
K \bar{C}_{y} & -I & 0 \\
0 & 0 & 0 \\
0 & 0 & 0
\end{array}\right], & \mathscr{P}=\left[\begin{array}{ccc}
P_{1} & 0 & 0 \\
-\alpha P_{2} K \bar{C}_{y} & \alpha P_{2} & 0 \\
P_{1} & 0 & 0 \\
-\alpha P_{2} K \bar{C}_{y} & \alpha P_{2} & 0
\end{array}\right] \\
\Lambda=\left[\begin{array}{ccc}
0 & 0 & 0 \\
0 & 0 & 0 \\
0 & 0 & -\gamma I
\end{array}\right], & \mathscr{S}=\left[\begin{array}{lll}
\bar{C} D_{z u} & D_{z w}
\end{array}\right]
\end{array}
$$

4) There exist matrices $P_{1}>0, P_{2}>0, G_{i}(i=1,2, \ldots, 6), H_{j}(j=1,2, \ldots, 8)$ and a sufficiently large scalar $\alpha>0$ such that 


$$
\Sigma=\left[\begin{array}{ccc}
\operatorname{Sym}\left(\mathscr{A}^{\mathrm{T}} \mathscr{G}\right)+\Lambda \mathscr{P}^{\mathrm{T}}-\mathscr{G}^{\mathrm{T}}+\mathscr{A}^{\mathrm{T}} \mathscr{H} \mathscr{S}^{\mathrm{T}} \\
* & -\mathscr{H}-\mathscr{H}^{\mathrm{T}} & 0 \\
* & * & -\gamma I
\end{array}\right]<0
$$

where

$$
\mathscr{G}=\left[\begin{array}{ccc}
G_{1} & G_{2} & G_{3} \\
-\alpha P_{2} K \bar{C}_{y} & \alpha P_{2} & 0 \\
G_{4} & G_{5} & G_{6} \\
-\alpha P_{2} K \bar{C}_{y} & \alpha P_{2} & 0
\end{array}\right], \quad \mathscr{H}=\left[\begin{array}{cccc}
H_{1} & H_{2} & H_{3} & H_{4} \\
0 & P_{2} & 0 & 0 \\
H_{5} & H_{6} & H_{7} & H_{8} \\
0 & 0 & 0 & P_{2}
\end{array}\right]
$$

Proof. Statements 1) and 2) constitute the well-known Bounded Real Lemma (Gahinet and Apkarian 1994). Then the whole proof can be obtained by using a procedure similar to the proofs of Theorems 1 and 2 in Shu and Lam (2009).

Remark 4. Statements 3) and 4) in Proposition 1 are the equivalent characterizations for the Bounded Real Lemma. Note that in these statements, the Lyapunov matrix $P_{1}$ has been separated from the system matrices, which may introduce more flexibility for parametrizing K. In particular, Statement 4) is obtained by introducing the slack matrices (Shu and Lam 2009; De Oliveira et al. 1999; Geromel and Colaneri 2006), which helps reduce the conservatism and improve the solvability of the iterative computation. Therefore, the conditions for the existence of a solution of Problem NFHPID to be presented later are based on Statement 4).

Lemma 1. (Xie et al. 1991) Given a real symmetric matrix $\mathscr{U}$, real matrices $\mathscr{V}, \mathscr{W}$ with appropriate dimensions, then

$$
\mathscr{U}+\mathscr{V} \mathscr{X} \mathscr{W}+\mathscr{W}^{T} \mathscr{X}^{\mathrm{T}} \mathscr{V}^{T}<0
$$

for all $\mathscr{X}$ satisfying $\mathscr{X}^{T} \mathscr{X} \leq I$ if and only if there exists a scalar $\mu>0$ such that

$$
\mathscr{U}+\mu \mathscr{V}^{T}+\mu^{-1} \mathscr{W}^{T} \mathscr{W}<0
$$

\section{Main results}

The aim of this paper is to design a non-fragile PID controller for the linear continuous-time system in (1) such that it is stable under the $H_{\infty}$ performance $\gamma$. Hence, the conditions for designing desired non-fragile controllers are obtained in the following theorems.

Theorem 1. For $\Delta K$ defined in (3) and (5), Problem NFHPID has a solution if there exist matrices $P_{1}>0$, $P_{2}>0, G_{i}(i=1,2, \ldots, 6), H_{j}(j=1,2, \ldots, 8), L, M$, a sufficiently large scalar $\alpha>0$ and a scalar $\varepsilon>0$ such that

$$
\bar{\Xi}(\alpha, M)=\left[\begin{array}{ccc}
\Xi & \varepsilon \xi P_{2} \overline{\mathscr{M}} \Upsilon^{\mathrm{T}} \mathscr{N}^{\mathrm{T}} \\
\varepsilon \overline{\mathscr{M}}^{\mathrm{T}} P_{2} \xi^{\mathrm{T}} & -\varepsilon I & 0 \\
\mathscr{N} \Upsilon & 0 & -\varepsilon I
\end{array}\right]<0
$$

where $\overline{\mathscr{M}}=\left[\mathscr{M}_{1} \mathscr{M}_{2} \mathscr{M}_{3}\right], F=\operatorname{diag}\left(F_{1}, F_{2}, F_{3}\right), \mathscr{N}=\operatorname{diag}\left(\mathscr{N}_{1}, \mathscr{N}_{2}, \mathscr{N}_{3}\right)$, 


$$
\Xi=\left[\begin{array}{cccccccc}
\Xi_{11} & \Xi_{12} & \Xi_{13} & P_{1}-G_{1}^{\mathrm{T}}+\bar{A}^{\mathrm{T}} H_{1} & \bar{A}^{\mathrm{T}} H_{2}+\bar{C}_{y}^{\mathrm{T}} L^{\mathrm{T}} & P_{1}-G_{4}^{\mathrm{T}}+\bar{A}^{\mathrm{T}} H_{3} & \bar{A}^{\mathrm{T}} H_{4} & \bar{C}^{\mathrm{T}} \\
* & \Xi_{22} & \bar{B}^{\mathrm{T}} G_{3}+G_{2}^{\mathrm{T}} \bar{B}_{w} & -G_{2}^{\mathrm{T}}+\bar{B}^{\mathrm{T}} H_{1} & \bar{B}^{\mathrm{T}} H_{2}-P_{2} & -G_{5}^{\mathrm{T}}+\bar{B}^{\mathrm{T}} H_{3} & \bar{B}^{\mathrm{T}} H_{4} & D_{z u}^{\mathrm{T}} \\
* & * & \Xi_{33} & -G_{3}^{\mathrm{T}}+\bar{B}_{w}^{\mathrm{T}} H_{1} & \bar{B}_{w}^{\mathrm{T}} H_{2} & -G_{6}^{\mathrm{T}}+\bar{B}_{w}^{\mathrm{T}} H_{3} & \bar{B}_{w}^{\mathrm{T}} H_{4} & D_{z w}^{\mathrm{T}} \\
* & * & * & -H_{1}-H_{1}^{\mathrm{T}} & -2 P_{2} & -H_{6}^{\mathrm{T}} & 0 & 0 \\
* & * & * & -2 P_{2} & -H_{6}^{\mathrm{T}} & 0 & 0 & \\
* & * & * & * & * & -H_{7}-H_{7}^{\mathrm{T}} & -H_{8} & 0 \\
* & * & * & * & * & * & -2 P_{2} & 0 \\
* & * & * & * & * & * & * & -\gamma I
\end{array}\right]
$$

$$
\begin{aligned}
& \Xi_{11}=\operatorname{Sym}\left(\bar{A}^{\mathrm{T}} G_{1}-2 \alpha M^{\mathrm{T}} L \bar{C}_{y}\right)+2 \alpha M^{\mathrm{T}} P_{2} M \\
& \Xi_{12}=\bar{A}^{\mathrm{T}} G_{2}+G_{1}^{\mathrm{T}} \bar{B}+2 \alpha \bar{C}_{y}^{\mathrm{T}} L^{\mathrm{T}} \\
& \Xi_{13}=\bar{A}^{\mathrm{T}} G_{3}+G_{1}^{\mathrm{T}} \bar{B}_{w} \\
& \Xi_{22}=\bar{B}^{\mathrm{T}} G_{2}+G_{2}^{\mathrm{T}} \bar{B}-2 \alpha P_{2} \\
& \Xi_{33}=\bar{B}_{w}^{\mathrm{T}} G_{3}+G_{3}^{\mathrm{T}} \bar{B}_{w}-\gamma I
\end{aligned}
$$

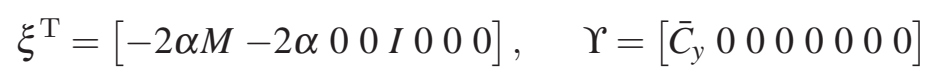

Under this condition, a non-fragile PID controller gain $K=\left[K_{p} K_{i} K_{d}\right]$ can be obtained as

$$
K=P_{2}^{-1} L
$$

Proof. Substituting $K+\Delta K$ into (15) and expanding it yield

$$
\begin{aligned}
& \bar{\Sigma}=\left[\begin{array}{cccc}
\Sigma_{11} & \Sigma_{12} & \Sigma_{13} & P_{1}-G_{1}^{\mathrm{T}}+\bar{A}^{\mathrm{T}} H_{1} \\
* & \Sigma_{22} & \bar{B}^{\mathrm{T}} G_{3}+G_{2}^{\mathrm{T}} \bar{B}_{w} & -G_{2}^{\mathrm{T}}+\bar{B}^{\mathrm{T}} H_{1} \\
* & * & \Sigma_{33} & -G_{3}^{\mathrm{T}}+\bar{B}_{w}^{\mathrm{T}} H_{1} \\
* & * & * & -H_{1}-H_{1}^{\mathrm{T}} \\
* & * & * & * \\
* & * & * & * \\
* & * & * & * \\
* & * & * & *
\end{array}\right. \\
& \left.\begin{array}{cccc}
\bar{A}^{\mathrm{T}} H_{2}+\bar{C}_{y}^{\mathrm{T}}(K+\Delta K)^{\mathrm{T}} P_{2} & P_{1}-G_{4}^{\mathrm{T}}+\bar{A}^{\mathrm{T}} H_{3} & \bar{A}^{\mathrm{T}} H_{4} & \bar{C}^{\mathrm{T}} \\
\bar{B}^{\mathrm{T}} H_{2}-P_{2} & -G_{5}^{\mathrm{T}}+\bar{B}^{\mathrm{T}} H_{3} & \bar{B}^{\mathrm{T}} H_{4} & D_{z u}^{\mathrm{T}} \\
\bar{B}_{w}^{\mathrm{T}} H_{2} & -G_{6}^{\mathrm{T}}+\bar{B}_{w}^{\mathrm{T}} H_{3} & \bar{B}_{w}^{\mathrm{T}} H_{4} & D_{z w}^{\mathrm{T}} \\
-H_{2} & -H_{5}^{\mathrm{T}}-H_{3} & -H_{4} & 0 \\
-2 P_{2} & -H_{6}^{\mathrm{T}} & 0 & 0 \\
* & -H_{7}-H_{7}^{\mathrm{T}} & -H_{8} & 0 \\
* & * & -2 P_{2} & 0 \\
* & * & * & -\gamma I
\end{array}\right]<0
\end{aligned}
$$


where

$$
\begin{aligned}
& \bar{\Sigma}_{11}=\operatorname{Sym}\left(\bar{A}^{\mathrm{T}} G_{1}\right)-2 \alpha \bar{C}_{y}^{\mathrm{T}}(K+\Delta K)^{\mathrm{T}} P_{2}(K+\Delta K) \bar{C}_{y} \\
& \bar{\Sigma}_{12}=\bar{A}^{\mathrm{T}} G_{2}+G_{1}^{\mathrm{T}} \bar{B}+2 \alpha \bar{C}_{y}^{\mathrm{T}}(K+\Delta K)^{\mathrm{T}} P_{2} \\
& \bar{\Sigma}_{13}=\bar{A}^{\mathrm{T}} G_{3}+G_{1}^{\mathrm{T}} \bar{B}_{w} \\
& \bar{\Sigma}_{22}=\bar{B}^{\mathrm{T}} G_{2}+G_{2}^{\mathrm{T}} \bar{B}-2 \alpha P_{2} \\
& \bar{\Sigma}_{33}=\bar{B}_{w}^{\mathrm{T}} G_{3}+G_{3}^{\mathrm{T}} \bar{B}_{w}-\gamma I
\end{aligned}
$$

According to Proposition 1, Problem SOF has a solution if and only if there exist matrices $P_{1}>0, P_{2}>0$, $G_{i}(i=1,2, \ldots, 6), H_{j}(j=1,2, \ldots, 8)$ and a sufficiently large scalar $\alpha>0$ such that (19) holds. Thus now we have to prove (19). It follows from $P_{2}>0$ and (18) that $L=P_{2} K$. The additive gain perturbations can be compactly expressed as $\Delta K=\mathscr{M} F \mathscr{N}$. By Schur complement equivalence, $\bar{\Xi}<0$ is equivalent to

$$
\Xi+\varepsilon \xi P_{2} \mathscr{M}\left(\xi P_{2} \mathscr{M}\right)^{\mathrm{T}}+\frac{1}{\varepsilon} \Upsilon^{\mathrm{T}} \mathscr{N}^{\mathrm{T}} \mathscr{N} \Upsilon<0
$$

It is noted that $F_{i}$ for $i=1,2,3$, satisfying (5) is equivalent to that $F$ satisfies $F^{\mathrm{T}} F \leq I$. From Lemma 2 and (20), it can be obtained that

$$
\Xi+\operatorname{Sym}\left(\xi P_{2} \mathscr{M} F \mathscr{N} \Upsilon\right)<0
$$

It follows from $\left((K+\Delta K) \bar{C}_{y}-M\right)^{\mathrm{T}} P_{2}\left((K+\Delta K) \bar{C}_{y}-M\right) \geq 0$ that

$$
\begin{aligned}
&-2 \alpha \bar{C}_{y}^{\mathrm{T}}(K+\Delta K)^{\mathrm{T}} P_{2}(K+\Delta K) \bar{C}_{y} \leq-\operatorname{Sym}\left(2 \alpha M^{\mathrm{T}} P_{2} K \bar{C}_{y}\right)+2 \alpha M^{\mathrm{T}} P_{2} M- \\
& \operatorname{Sym}\left(2 \alpha M^{\mathrm{T}} P_{2} \Delta K \bar{C}_{y}\right)
\end{aligned}
$$

Therefore by observing $\bar{\Sigma}_{11}$ and $\Xi_{11}$, we have

$$
\bar{\Sigma} \leq \Xi+\operatorname{Sym}\left(\xi P_{2} \mathscr{M} F \mathscr{N} \Upsilon\right)<0
$$

This concludes that Problem SOF has a solution which also means that Problem NFHPID has a solution if (16) holds. The proof is completed.

The multiplicative gain perturbations can be written as $\Delta K=K \tilde{\mathscr{M}} F \mathscr{N}$ compactly where $\tilde{\mathscr{M}}=\operatorname{diag}\left(\tilde{\mathscr{M}}_{1}\right.$, $\left.\tilde{\mathscr{M}}_{2}, \tilde{\mathscr{M}}_{3}\right)$. Then by following a similar line from the proof in Theorem 1 , the condition for designing desired non-fragile controllers is obtained as follows.

Theorem 2. For $\Delta K$ defined in (4) and (5), Problem NFHPID has a solution if there exist matrices $P_{1}>0$, $P_{2}>0, G_{i}(i=1,2, \ldots, 6), H_{j}(j=1,2, \ldots, 8), L, M$, a sufficiently large scalar $\alpha>0$ and a scalar $\varepsilon>0$ such that

$$
\tilde{\Xi}(\alpha, M)=\left[\begin{array}{ccc}
\Xi & \varepsilon \xi L \tilde{\mathscr{M}} \Upsilon^{\mathrm{T}} \mathscr{N}^{\mathrm{T}} \\
\varepsilon \tilde{\mathscr{M}}^{\mathrm{T}} L^{\mathrm{T}} \xi^{\mathrm{T}} & -\varepsilon I & 0 \\
\mathscr{N} \Upsilon & 0 & -\varepsilon I
\end{array}\right]<0
$$

where $F, \mathscr{N}, \Xi, \xi$ and $\Upsilon$ are defined in Theorem 1. Under this condition, a non-fragile PID controller gain $K=\left[K_{p} K_{i} K_{d}\right]$ can be obtained as

$$
K=P_{2}^{-1} L
$$


Remark 5. Additive gain perturbations have been considered in Theorem 1 while the multiplicative gain perturbations have been considered in Theorem 2. If the controller gain perturbations are neglected, that is $\Delta K \equiv 0$, the condition (16) in Theorem 1 and the condition (21) in Theorem 2 are both reduced to that

$$
\Xi(\alpha, M)<0
$$

It is worth mentioning that (23) is a sufficient condition for the existence of a solution for Problem NFHPID (which is also a necessary and sufficient condition for the existence of a solution for Problem SOF) when $\Delta K \equiv 0$. Hence one can design a nominal PID controller by computing this condition directly in the algorithm.

Remark 6. In this paper, we focus on the PID controller of centralised type whose structure is more complicated. By observing its controller gains $K_{\mathrm{P}}, K_{\mathrm{I}}, K_{\mathrm{D}} \in \mathbb{R}^{r \times m}$, the number of parameters to find is $3 \mathrm{rm}$. Particularly, if $r=m$, it is possible to design a decentralised PID controller of which gains have the following structure:

$$
K=\left[K_{\mathrm{P}} K_{\mathrm{I}} K_{\mathrm{D}}\right]=\left[\begin{array}{cccccccccccc}
k_{\mathrm{P}_{1}} & 0 & \cdots & 0 & k_{\mathrm{I}_{1}} & 0 & \cdots & 0 & k_{\mathrm{D}_{1}} & 0 & \cdots & 0 \\
0 & k_{\mathrm{P}_{2}} & \cdots & 0 & 0 & k_{\mathrm{I}_{2}} & \cdots & 0 & 0 & k_{\mathrm{D}_{2}} & \cdots & 0 \\
0 & 0 & \ddots & 0 & 0 & 0 & \ddots & 0 & 0 & 0 & \ddots & 0 \\
0 & 0 & \cdots & k_{\mathrm{P}_{r}} & 0 & 0 & \cdots & k_{\mathrm{I}_{r}} & 0 & 0 & \cdots & k_{\mathrm{D}_{r}}
\end{array}\right] \in \mathbb{R}^{r \times 3 m}
$$

and the number of parameters to find is $3 r$. It is obvious that the centralised PID controller requires many more parameters to tune compared to the decentralised type. Furthermore, if $P_{2}$ and $L$ defined in previous Theorems 1 and 2 have the following structures:

$$
P_{2}=\left[\begin{array}{cccc}
p_{21} & 0 & \cdots & 0 \\
0 & p_{22} & \cdots & 0 \\
0 & 0 & \ddots & 0 \\
0 & 0 & \cdots & p_{2 r}
\end{array}\right], \quad L=\left[\begin{array}{cccccccccccc}
l_{11} & 0 & \cdots & 0 & l_{21} & 0 & \cdots & 0 & l_{31} & 0 & \cdots & 0 \\
0 & l_{12} & \cdots & 0 & 0 & l_{22} & \cdots & 0 & 0 & l_{32} & \cdots & 0 \\
0 & 0 & \ddots & 0 & 0 & 0 & \ddots & 0 & 0 & 0 & \ddots & 0 \\
0 & 0 & \cdots & l_{1 r} & 0 & 0 & \cdots & l_{2 r} & 0 & 0 & \cdots & l_{3 r}
\end{array}\right]
$$

where $p_{2 j}, l_{1 j}, l_{2 j}$ and $l_{3 j}$ for $j=1,2, \ldots, r$, are scalars, we can design decentralised PID controllers with gains as shown in (24) under similar conditions (16), (21), and (23), though the controllers may not achieve better performances than the centralised type.

Remark 7. Since Problem NFHPID has been transformed to Problem SOF, one can solve the conditions (16), (21) and (23) by constructing an LMI based iterative algorithm which is similar to Algorithm 1 in Shu and Lam (2009). In addition, it is worth pointing out that the initial values in the algorithm can be optimised to improve the solvability of it. For details about the algorithm and the optimisation of initial values, one may refer to (Shu and Lam 2009).

\section{Illustrative examples}

To illustrate the use of the proposed approaches, simulations are conducted on the design of PID controller in this section. Examples 1 is used to verify the effectiveness of our proposed approach and the PID controllers for the system in Zheng et al. (2002) are designed. In Examples 2 and 3, the non-fragility of PID controllers under two types of gain perturbations is verified and comparisons of them are provided as well. For convenience of illustration, matrix $\mathscr{T}=\tau I$ where $\tau=0.015915$ is used in the simulations. The value of the low-pass filter time constant corresponds to a cut-off frequency of $10 \mathrm{~Hz}$. 


\subsection{Example 1}

For the purpose of comparison, we consider and discuss the aircraft (AC) state-space model (Zheng et al. 2002) with the following parameters:

$$
\begin{aligned}
& A=\left[\begin{array}{cccccc}
-0.0266 & -36.6170 & -18.8970 & -32.0900 & 3.2509 & -0.7626 \\
0.0001 & -1.8997 & 0.9831 & -0.0007 & -0.1708 & -0.0050 \\
0.0123 & 11.7200 & -2.6316 & 0.0009 & -31.6040 & 22.3960 \\
0 & 0 & 1.0000 & 0 & 0 & 0 \\
0 & 0 & 0 & 0 & -30.0000 & 0 \\
0 & 0 & 0 & 0 & 0 & -30.0000
\end{array}\right], B=\left[\begin{array}{cc}
0 & 0 \\
0 & 0 \\
0 & 0 \\
0 & 0 \\
30 & 0 \\
0 & 30
\end{array}\right]
\end{aligned}
$$

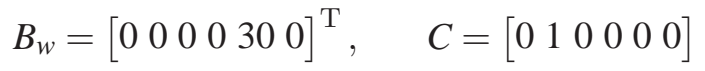

$$
\begin{aligned}
& C_{y}=\left[\begin{array}{llllll}
0 & 1 & 0 & 0 & 0 & 0 \\
0 & 0 & 0 & 1 & 0 & 0
\end{array}\right], \quad D_{z u}=\left[\begin{array}{ll}
1 & 1
\end{array}\right], \quad D_{z w}=[0]
\end{aligned}
$$

Simulation results on the design of PID controllers for the AC model are summarized in Table 1. The result solved by the approach of (Zheng et al. 2002) is reproduced here as well.

For this model, centralised and decentralised PID controllers have been designed by our proposed approaches without perturbations considered. It can be seen from Table 1 that the proposed centralised approach achieves a better performance than the proposed decentralised one. Besides, both our approach (centralised) and the approach of (Zheng et al. 2002) achieve the same performance level $\gamma=1.0000$. It can be observed that many closed-loop poles obtained from their method are very close to the stability boundary which is undesirable. Simulation results have verified the effectiveness our proposed approaches.

Table 1. PID-controller and $H_{\infty}$ performance of AC model.

\begin{tabular}{|c|c|c|c|}
\hline Approach & Controller gains & Closed-loop poles & Performance \\
\hline $\begin{array}{l}\text { Proposed } \\
\text { (Centralised) }\end{array}$ & $\begin{array}{l}K_{\mathrm{P}}=\left[\begin{array}{ll}26.203 & -6.0394 \\
2.5499 & -5.9430\end{array}\right] \\
K_{\mathrm{I}}=\left[\begin{array}{cc}16.413 & -1.7124 \\
-0.55271 & -5.4532\end{array}\right] \\
K_{\mathrm{D}}=\left[\begin{array}{cc}6.8425 & -6.4368 \\
-0.95989 & 0.60049\end{array}\right]\end{array}$ & $\begin{array}{c}-69.230 \\
-42.073 \pm j 39.294 \\
-13.895 \pm j 16.880 \\
-0.024923 \\
-0.59398 \pm j 0.31582 \\
-3.9208 \pm 3.2282\end{array}$ & 1.0000 \\
\hline $\begin{array}{l}\text { Proposed } \\
\text { (Decentralised) }\end{array}$ & $\begin{array}{l}K_{\mathrm{P}}=\left[\begin{array}{cc}1.4115 & 0 \\
0 & -3.2398\end{array}\right] \\
K_{\mathrm{I}}=\left[\begin{array}{cc}9.0245 & 0 \\
0 & -13.3225\end{array}\right] \\
K_{\mathrm{D}}=\left[\begin{array}{cc}0.34970 & 0 \\
0 & -0.47470\end{array}\right]\end{array}$ & $\begin{array}{c}-74.639 \\
-60.770 \\
-31.870 \\
-8.8086 \pm j 21.264 \\
-2.1328 \pm j 4.9966 \\
-0.51820 \pm j 0.69770 \\
-0.02480\end{array}$ & 1.5240 \\
\hline (Zheng et al. 2002) & $\begin{array}{l}K_{\mathrm{P}}=\left[\begin{array}{cc}422.17 & 221.64 \\
-188.84 & -104.44\end{array}\right] \\
K_{\mathrm{I}}=\left[\begin{array}{ll}0.38450 & -0.50190 \\
0.10680 & -0.33730\end{array}\right] \\
K_{\mathrm{D}}=\left[\begin{array}{cc}48.030 & -31.850 \\
-19.310 & 8.8000\end{array}\right]\end{array}$ & $\begin{array}{c}-0.00010 \\
-0.0020000 \\
-0.0050000 \\
-0.72000 \\
-19.910 \\
-48.020 \pm j 77.790 \\
-191.00\end{array}$ & 1.0000 \\
\hline
\end{tabular}




\subsection{Example 2}

In this example, we consider a system which is the longitudinal motion of a VTOL helicopter (HE1) (Leibfritz 2003) with the following given model data:

$$
A=\left[\begin{array}{cccc}
-0.0366 & 0.0271 & 0.0188 & -0.4555 \\
0.0482 & -1.01 & 0.0024 & -4.0208 \\
0.1002 & 0.3681 & -0.707 & 1.42 \\
0 & 0 & 1 & 0
\end{array}\right], \quad B=\left[\begin{array}{cc}
0.4422 & 0.1761 \\
3.5446 & -7.5922 \\
-5.52 & 4.49 \\
0 & 0
\end{array}\right], \quad C_{y}^{\mathrm{T}}=\left[\begin{array}{l}
0 \\
1 \\
0 \\
0
\end{array}\right]
$$

We choose $C=\left[\begin{array}{llll}\sqrt{2} & 0 & 0 & 0\end{array}\right]^{\mathrm{T}}, B_{w}=\left[\begin{array}{llll}0.0468 & 0 & 0.0437 & 0\end{array}\right]^{\mathrm{T}}, D_{z w}=0$ and $D_{z u}=\left[\begin{array}{lll}\sqrt{2} & 2 & 0\end{array}\right]$, respectively, so that $C_{y} B_{w}=0$ (Assumption 1 in Zheng et al. (2002)) holds. To verify the non-fragility of PID controllers, gain perturbation parameters are given as follows.

- Norm-bounded additive form: $\mathscr{M}_{1}=[-0.00016782-0.00081491]^{\mathrm{T}}, \mathscr{M}_{2}=[-0.00062758-$ $0.0022820]^{\mathrm{T}}, \mathscr{M}_{3}=\left[\begin{array}{ll}0.000049118 & 0.000062182\end{array}\right]^{\mathrm{T}}, \mathscr{N}_{1}=\mathscr{N}_{2}=\mathscr{N}_{3}=1$.

- Norm-bounded multiplicative form: $\tilde{\mathscr{M}}_{1}=-0.057576, \tilde{\mathscr{M}}_{2}=0.0012543, \tilde{\mathscr{M}}_{3}=-0.048352, \mathscr{N}_{1}=$ $\mathscr{N}_{2}=\mathscr{N}_{3}=1$.

Simulation results on the design of PID controllers for the HE1 model are summarized in Table 2. The result solved by the approach of (Zheng et al. 2002) is also provided here. Note that the non-fragile additive and multiplicative PID controllers have their guaranteed $H_{\infty}$ performance values given by 0.85822 and 0.56273 , respectively.

In order to verify the non-fragility of controllers, 50 samples of $F_{i} \in \mathbb{R}$ for $i=1,2,3$, are randomly generated from the standard uniform distribution on the interval $(-1,1)$. Under the gain perturbations, stability and $H_{\infty}$ performance results of this model with PID controllers in Table 2 are given in Tables 3 and 4. Also, the perturbed poles of closed-loop system under additive and multiplicative perturbations are presented in Figures 1 and 2. It can be seen from Tables 3 and 4, and Figures 1 and 2 that under the gain perturbations, the PID controllers designed by our proposed approach are always stabilizing but the one designed by (Zheng et al. 2002) is not. Furthermore, from the view of $H_{\infty}$ performance, the results of PID controllers designed by our proposed approach in Table 3 show that the non-fragile PID controller achieves higher reliability than the nominal PID controller. This is because through our approach, the non-fragile PID controllers have the guaranteed $H_{\infty}$ performances (shown in Table 2 as bracketed numbers) while the PID controllers designed by the proposed nominal approach and that of (Zheng et al. 2002) do not have.

\subsection{Example 3}

Consider the system (NN17) borrowed from (Leibfritz 2003) as in (1) with the following parameters:

$$
\begin{gathered}
A=\left[\begin{array}{ccc}
0 & -1 & 2 \\
1 & -2 & 3 \\
0 & 1 & 0
\end{array}\right], \quad B=\left[\begin{array}{cc}
1 & 0 \\
0 & 0 \\
0 & -1
\end{array}\right], \quad B_{w}=\left[\begin{array}{c}
1 \\
-1 \\
0
\end{array}\right], \quad C_{y}=\left[\begin{array}{lll}
1 & 0 & 0
\end{array}\right] \\
D_{z u}=\left[\begin{array}{ll}
0 & 1 \\
0 & 0
\end{array}\right], \quad D_{z w}=\left[\begin{array}{l}
0 \\
0
\end{array}\right], \quad C=\left[\begin{array}{lll}
1 & 0 & 1 \\
1 & 0 & 1
\end{array}\right]
\end{gathered}
$$

Similarly, the perturbation parameters are given as follows.

- Norm-bounded additive form: $\mathscr{M}_{1}=\left[\begin{array}{lll}0.064950 & 0.052817\end{array}\right]^{\mathrm{T}}, \mathscr{M}_{2}=\left[\begin{array}{lll}0.023166 & -0.085149\end{array}\right]^{\mathrm{T}}, \mathscr{M}_{3}$ $=\left[\begin{array}{ll}-0.048706 & 0.13497\end{array}\right]^{\mathrm{T}}, \mathscr{N}_{1}=\mathscr{N}_{2}=\mathscr{N}_{3}=1$.

- Norm-bounded multiplicative form: $\tilde{\mathscr{M}}_{1}=0.019154, \tilde{\mathscr{M}}_{2}=-0.018656, \tilde{\mathscr{M}}_{3}=-0.020334, \mathscr{N}_{1}=$ $\mathscr{N}_{2}=\mathscr{N}_{3}=1$. 
Since the assumption $C_{y} B_{w}=0$ (Assumption 1 in Zheng et al. (2002)) does not hold for this system, the approach of (Zheng et al. 2002) is not applicable here when the $H_{\infty}$ performance is considered. For comparative purpose, we use the stabilization approach of (Zheng et al. 2002) to obtain the controller and obtain its nominal performance (guaranteed performance cannot be computed since the assumption $C_{y} B_{w}=0$ is not satisfied in their method). Simulation results on our three design conditions and the stabilization approach of (Zheng et al. 2002) are given in Table 5. Note that for the non-fragile additive and multiplicative PID controllers, the guaranteed $H_{\infty}$ performances are 14.029 and 14.762 , respectively. Also the $H_{\infty}$ performance value of closed-loop system with PID controller by the approach of (Zheng et al. 2002) is 57.692. 50 samples of $F_{i} \in \mathbb{R}$ for $i=1,2,3$, are randomly generated from the standard uniform distribution on the interval $(-1,1)$. Under the gain perturbations, stability and $H_{\infty}$ performance results of this model with the PID controllers are shown in Tables 6 and 7. The perturbed poles of closed-loop system are presented in Figures 3 and 4 .

Simulation results in Tables 6 and 7, and Figures 3 and 4 show that the PID controllers designed by

Table 2. PID-controller and $H_{\infty}$ performance of HE1 model.

\begin{tabular}{|c|c|c|c|}
\hline Approach & Controller gains & Closed-loop poles & $\begin{array}{l}\text { Nominal } \\
\text { Performance } \\
\text { (Guaranteed } \\
\text { Performance) }\end{array}$ \\
\hline $\begin{array}{l}\text { Proposed } \\
\text { (No perturbation) }\end{array}$ & $\begin{array}{l}K_{\mathrm{P}}=\left[\begin{array}{l}1.0864 \\
5.0973\end{array}\right] \\
K_{\mathrm{I}}=\left[\begin{array}{c}0.032433 \\
0.10730\end{array}\right] \\
K_{\mathrm{D}}=\left[\begin{array}{c}-0.39615 \\
4.6688\end{array}\right]\end{array}$ & $\begin{array}{c}-2413.1 \\
-0.60537 \pm j 0.86869 \\
-0.22182 \pm j 0.15459 \\
-0.019836\end{array}$ & 0.22136 \\
\hline $\begin{array}{l}\text { Proposed } \\
\text { (Additive perturbation) }\end{array}$ & $\begin{array}{l}K_{\mathrm{P}}=\left[\begin{array}{l}3.5878 \\
19.265\end{array}\right] \\
K_{\mathrm{I}}=\left[\begin{array}{c}0.28716 \\
2.2423\end{array}\right] \\
K_{\mathrm{D}}=\left[\begin{array}{c}-0.071181 \\
10.787\end{array}\right]\end{array}$ & $\begin{array}{c} \\
-5357.2 \\
-0.096218 \\
-0.48116 \pm j 0.51279 \\
-0.53359 \\
-0.72961\end{array}$ & $\begin{array}{c}0.14714 \\
(0.85822)\end{array}$ \\
\hline $\begin{array}{l}\text { Proposed } \\
\text { (Multiplicative perturbation) }\end{array}$ & $\begin{array}{l}K_{\mathrm{P}}=\left[\begin{array}{l}0.093724 \\
0.49328\end{array}\right] \\
K_{\mathrm{I}}=\left[\begin{array}{c}0.068802 \\
0.27793\end{array}\right] \\
K_{\mathrm{D}}=\left[\begin{array}{c}0.29087 \\
1.6678\end{array}\right]\end{array}$ & $\begin{array}{c}-797.74 \\
-0.27045 \\
-0.24447 \pm j 0.54723 \\
-0.16483 \pm j 0.33556\end{array}$ & $\begin{array}{c}0.22660 \\
(0.56273)\end{array}$ \\
\hline (Zheng et al. 2002) & $\begin{array}{l}K_{\mathrm{P}}=\left[\begin{array}{c}0.62414 \\
-0.52290\end{array}\right] \\
K_{\mathrm{I}}=\left[\begin{array}{c}-0.024578 \\
-0.85139\end{array}\right] \\
K_{\mathrm{D}}=\left[\begin{array}{c}-0.0069242 \\
-0.13600\end{array}\right]\end{array}$ & $\begin{array}{c}-641.99 \\
-1.0001 \pm j 1.3717 \\
-0.21001 \pm j 0.015734\end{array}$ & 0.24570 \\
\hline
\end{tabular}
$(2+2$

ion 1 in Zheng et al. (2002)) does not hold for
abuch of (Zheng et al. 2002) to obtain the cont
results on our three design conditions and the
ances are 14.029 and 14.762, respectively. Al
D controller by the approach of (Zheng et al.
7. The perturbed poles of closed-loop system
Figures 3 and 4 show that the PID controllers 
Table 3. Stability and $H_{\infty}$ performance of HE1 model (Additive perturbation) over 50 trials.

\begin{tabular}{lccccc}
\hline \multirow{2}{*}{ Approach } & Stability & \multicolumn{5}{c}{ Performance } \\
\cline { 3 - 6 } & $100 \%$ & 0.16960 & 6.8316 & 0.82261 & 1.3078 \\
\hline $\begin{array}{l}\text { Proposed } \\
\text { (No perturbation) }\end{array}$ & $100 \%$ & 0.14533 & 0.14831 & 0.14724 & 0.00072563 \\
\hline $\begin{array}{l}\text { Proposed } \\
\text { (Additive perturbation) }\end{array}$ & $52 \%$ & 0.20088 & 0.24839 & 0.21635 & 0.011695 \\
\hline (Zheng et al. 2002) & & & & & Mean \\
\hline
\end{tabular}

Table 4. Stability and $H_{\infty}$ performance of HE1 model (Multiplicative perturbation) over 50 trials.

\begin{tabular}{lccccc}
\hline \multirow{2}{*}{ Approach } & Stability & \multicolumn{4}{c}{ Performance } \\
\cline { 3 - 6 } & & Min & Max & Mean & Standard deviation \\
\hline $\begin{array}{l}\text { Proposed } \\
\text { (No perturbation) }\end{array}$ & $100 \%$ & 0.22139 & 0.22139 & 0.22139 & 0 \\
\hline $\begin{array}{l}\text { Proposed } \\
\text { (Multiplicative perturbation) }\end{array}$ & $100 \%$ & 0.22094 & 0.23440 & 0.22688 & 0.0038731 \\
\hline \begin{tabular}{l} 
(Zheng et al. 2002) \\
\hline
\end{tabular} & $59 \%$ & 0.24553 & 0.25014 & 0.24638 & 0.00085381 \\
\hline
\end{tabular}

our proposed approaches are always stabilizing under the gain perturbations. Although the PID controller designed by the approach of (Zheng et al. 2002) is always stabilizing under the 50 randomly generated additive and multiplicative perturbations despite stability robustness is not theoretically ensured, the achieved $H_{\infty}$ performance values are much greater than that of the PID controllers designed by our proposed approaches. From the view of $H_{\infty}$ performance, the non-fragile PID controllers can achieve smaller values and they are more reliable as compared with the nominal PID controller. In Table 7, the $H_{\infty}$ performance values of closed-loop system with PID controller do not vary under multiplicative gain perturbation. This result can be illustrated intuitively by Figure 5 which presents the closed-loop frequency response with PID controller synthesized for tackling multiplicative perturbation. It shows that at frequency $0 \mathrm{rad} / \mathrm{s}$, the peak gain is achieved and is analytically given by

$$
\frac{\sqrt{2 K_{\mathrm{I} 1}^{2}-22 K_{\mathrm{I} 1} K_{\mathrm{I} 2}+73 K_{\mathrm{I} 2}^{2}}}{\left|3 K_{\mathrm{I} 1}+K_{\mathrm{I} 2}\right|}
$$

where $K_{\mathrm{I}}=\left[\begin{array}{ll}K_{\mathrm{I} 1} & K_{\mathrm{I} 2}\end{array}\right]^{\mathrm{T}}$. With the values of $K_{\mathrm{I}}$, the $H_{\infty}$ norm is $11.594(21.285 \mathrm{~dB})$. It should be noted that the multiplicative perturbation structure in (4) does not affect the $H_{\infty}$ norm in this example.

Furthermore, in order to verify the decentralized PID controllers designed by our approach, we consider the same system as in (25) but with a different output matrix:

$$
C_{y}=\left[\begin{array}{lll}
1 & 0 & 0 \\
0 & 0 & 1
\end{array}\right]
$$

Denote this system as the modified NN17 (MNN17) model. Notice that the assumption $C_{y} B_{w}=0$ required in (Zheng et al. 2002) is again not satisfied. The perturbation parameters are given as follows.

- Norm-bounded additive form:

$$
\begin{array}{ll}
\mathscr{M}_{1}=\left[\begin{array}{cc}
0.54760 & 0 \\
0 & -0.67936
\end{array}\right] & \mathscr{M}_{2}=\left[\begin{array}{cc}
-0.62385 & 0 \\
0 & 0.20328
\end{array}\right] \\
\mathscr{M}_{3}=\left[\begin{array}{cc}
0.69881 & 0 \\
0 & 0.11295
\end{array}\right] & \mathscr{N}_{1}=\mathscr{N}_{2}=\mathscr{N}_{3}=\left[\begin{array}{cc}
1.0000 & 0 \\
0 & 1.0000
\end{array}\right]
\end{array}
$$




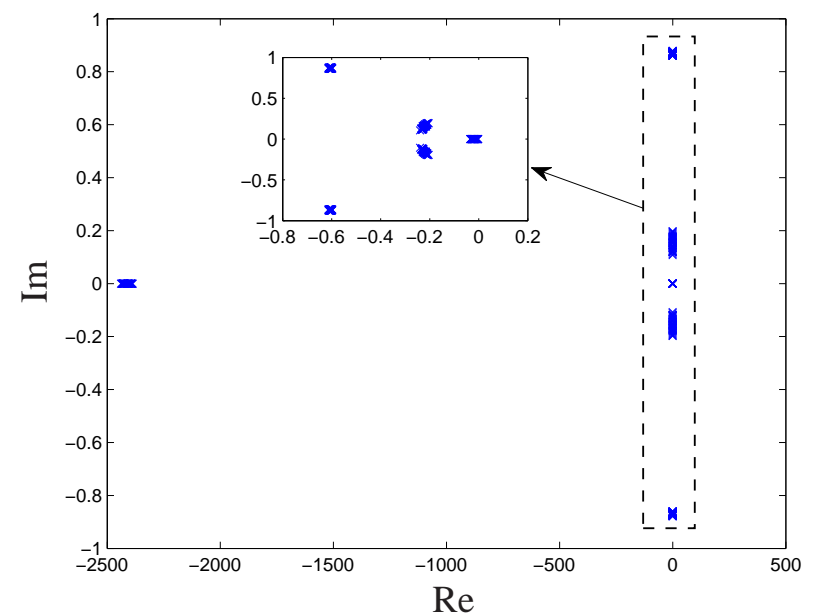

(a) Perturbed closed-loop poles with proposed nominal PID controller.

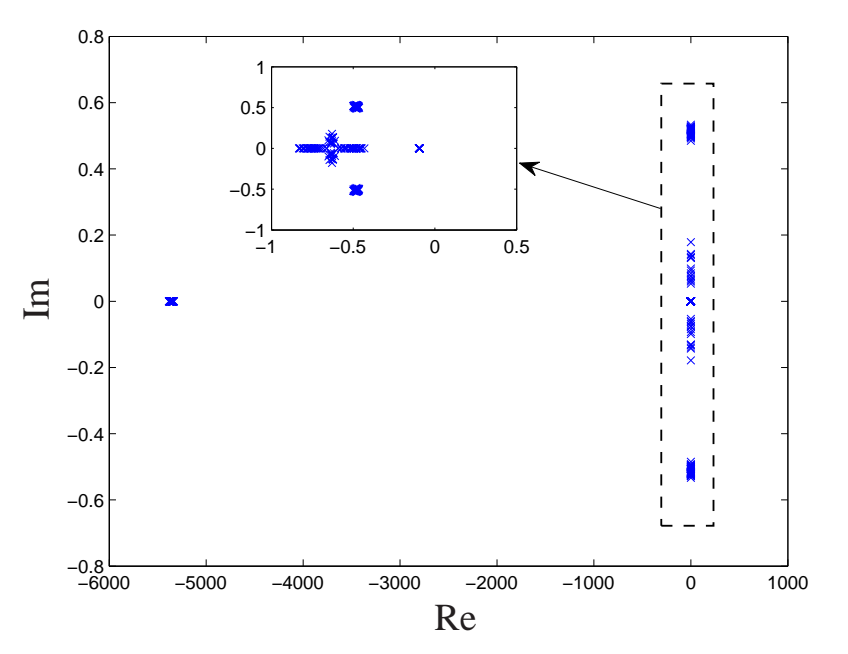

(b) Perturbed closed-loop poles with proposed non-fragile (additive) PID controller.

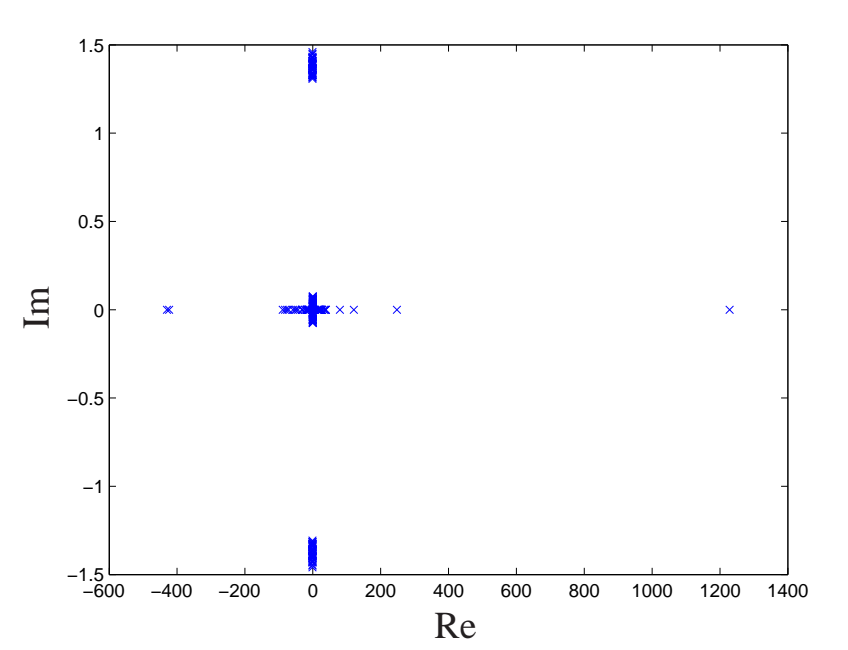

(c) Perturbed closed-loop poles with PID controller (Zheng et al. 2002).
Figure 1. Perturbed closed-loop poles (HE1) under additive perturbation.

(c) Perturbed closed-loop poles with PID controller (Zheng et al. 2002).
Figure 1. Perturbed closed-loop poles (HE1) under additive perturbation.

$\operatorname{Re}$

.




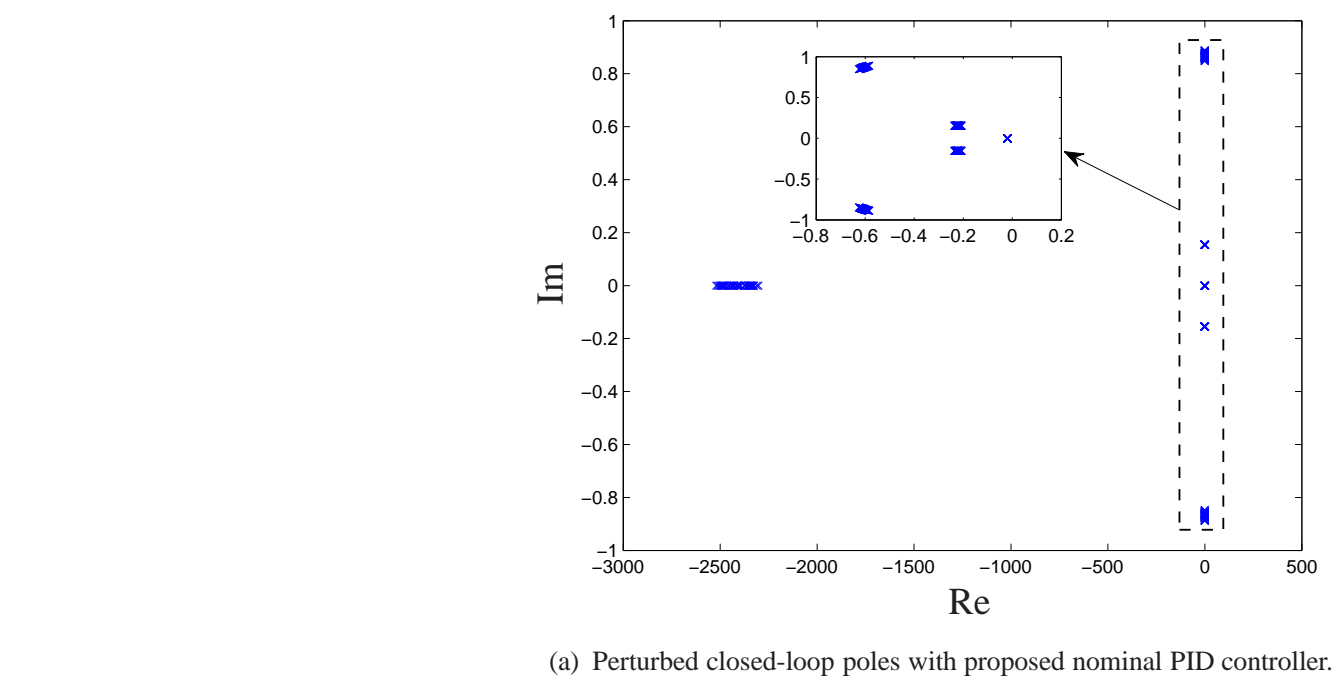

(a) Perturbed closed-loop poles with proposed nominal PID controller.

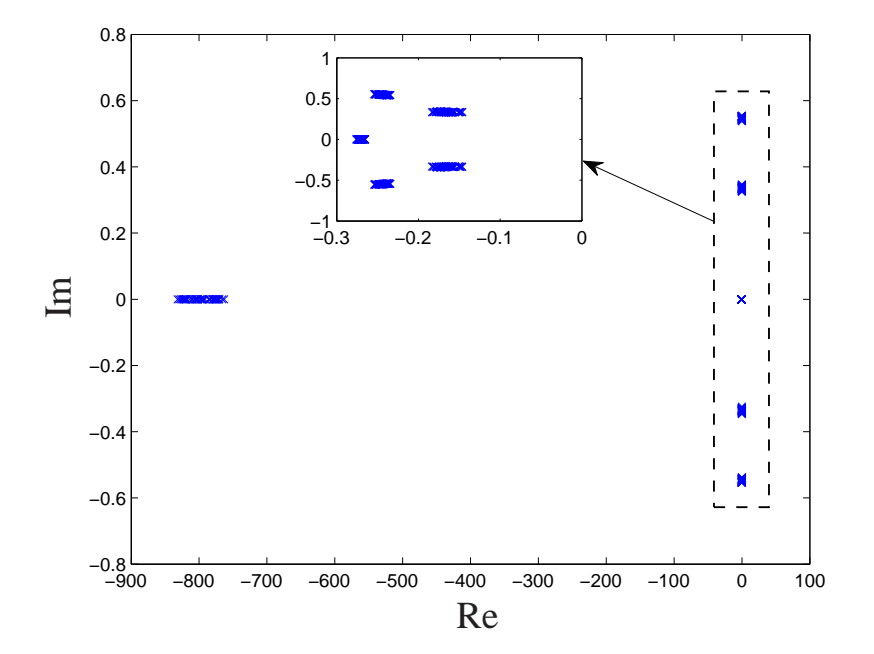

(b) Perturbed closed-loop poles with proposed non-fragile (multiplicative) PID controller.

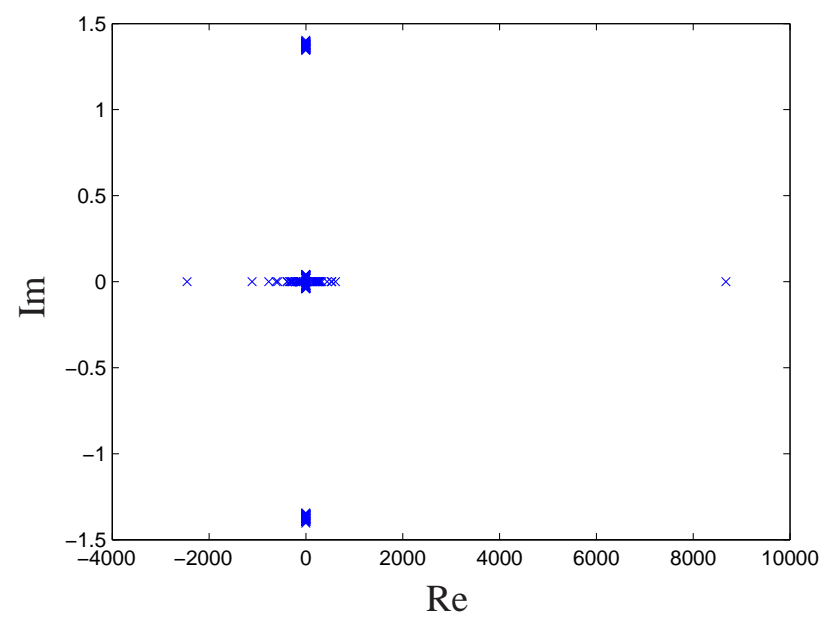

(c) Perturbed closed-loop poles with PID controller (Zheng et al. 2002).

Figure 2. Perturbed closed-loop poles (HE1) under multiplicative perturbation. 
Table 5. PID-controller and $H_{\infty}$ performance of NN17 model.

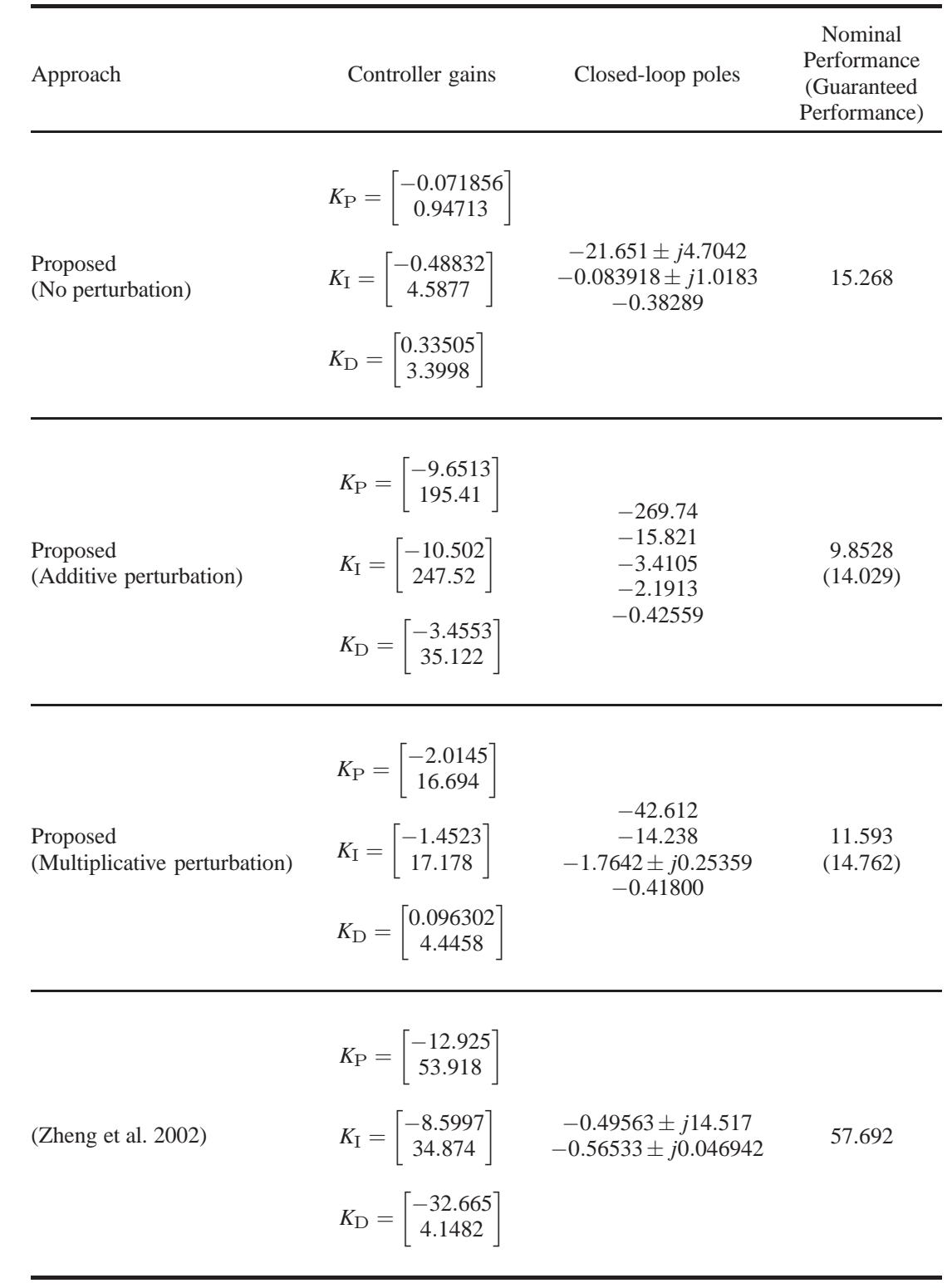

Table 6. Stability and $H_{\infty}$ performance of NN17 model (Additive perturbation) over 50 trials.

\begin{tabular}{lccccc}
\hline \multirow{2}{*}{ Approach } & \multirow{2}{*}{ Stability } & \multicolumn{4}{c}{ Performance } \\
\cline { 3 - 6 } & Min & Max & Mean & Standard deviation \\
\hline $\begin{array}{l}\text { Proposed } \\
\text { (No perturbation) }\end{array}$ & $100 \%$ & 12.889 & 18.139 & 15.419 & 1.3094 \\
\hline $\begin{array}{l}\text { Proposed } \\
\text { (Additive perturbation) }\end{array}$ & $100 \%$ & 9.8500 & 9.8553 & 9.8528 & 0.0014786 \\
\hline (Zheng et al. 2002) & $100 \%$ & 57.274 & 58.242 & 57.703 & 0.25994 \\
\hline
\end{tabular}

- Norm-bounded multiplicative form:

$$
\begin{aligned}
& \tilde{\mathscr{M}}_{1}=\left[\begin{array}{cc}
-0.011154 & 0 \\
0 & 0.0030439
\end{array}\right] \quad \tilde{\mathscr{M}}_{2}=\left[\begin{array}{cc}
-0.027305 & 0 \\
0 & 0.030748
\end{array}\right] \\
& \tilde{\mathscr{M}}_{3}=\left[\begin{array}{cc}
-0.0072509 & 0 \\
0 & -0.030303
\end{array}\right] \quad \mathscr{N}_{1}=\mathscr{N}_{2}=\mathscr{N}_{3}=\left[\begin{array}{cc}
1.0000 & 0 \\
0 & 1.0000
\end{array}\right]
\end{aligned}
$$

Proposed

$$
\begin{aligned}
K_{\mathrm{I}} & =\left[\begin{array}{c}
-0.48832 \\
4.5877
\end{array}\right] \\
K_{\mathrm{D}} & =\left[\begin{array}{c}
0.33505 \\
3.3998
\end{array}\right]
\end{aligned}
$$

$$
\begin{aligned}
& -0.38289 \\
& \text { - }
\end{aligned}
$$




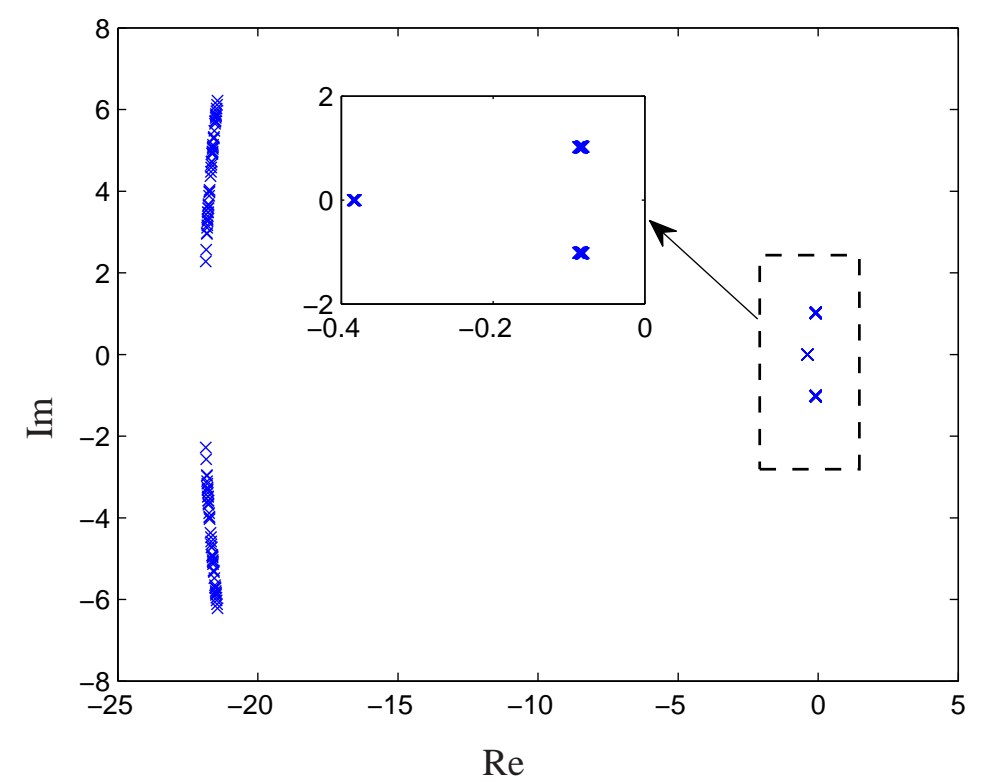

(a) Perturbed closed-loop poles with proposed nominal PID controller.

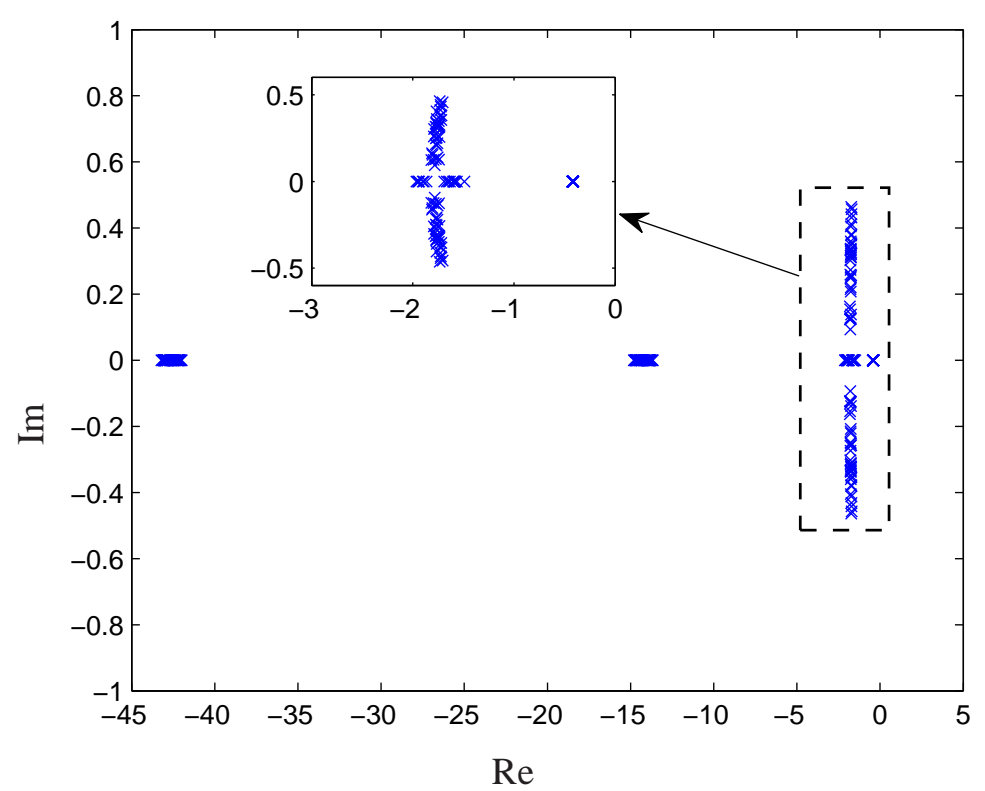

(b) Perturbed closed-loop poles with proposed non-fragile (multiplicative) PID controller.

Figure 4. Perturbed closed-loop poles (NN17) under multiplicative perturbation.

Simulation results on the decentralized PID controllers are given in Table 8 . The guaranteed $H_{\infty}$ performances are 7.3206 and 8.2321 for the decentralised non-fragile additive and multiplicative PID controllers, respectively. Similarly, 50 samples of diagonal matrices $F_{i} \in \mathbb{R}^{2 \times 2}$ for $i=1,2,3$, are randomly generated from the standard uniform distribution on the interval $(-1,1)$. Under the gain perturbations, stability and $H_{\infty}$ performance results of this model with the decentralised PID controllers are shown in Tables 9 and 10. The perturbed poles of the closed-loop systems are presented in Figures 6 and 7.

The results in Tables 9 and 10, and Figures 6 and 7 show that the decentralised non-fragile PID controllers are always stabilizing though the $H_{\infty}$ performance values of the corresponding closed-loop systems are greater. They have verified the reliability of the decentralised PID controllers designed by our approach. In all, these simulation results indicate that the non-fragile PID controllers have better gain perturbation 
Table 9. Stability and $H_{\infty}$ performance of MNN17 model (Additive perturbation) over 50 trials.

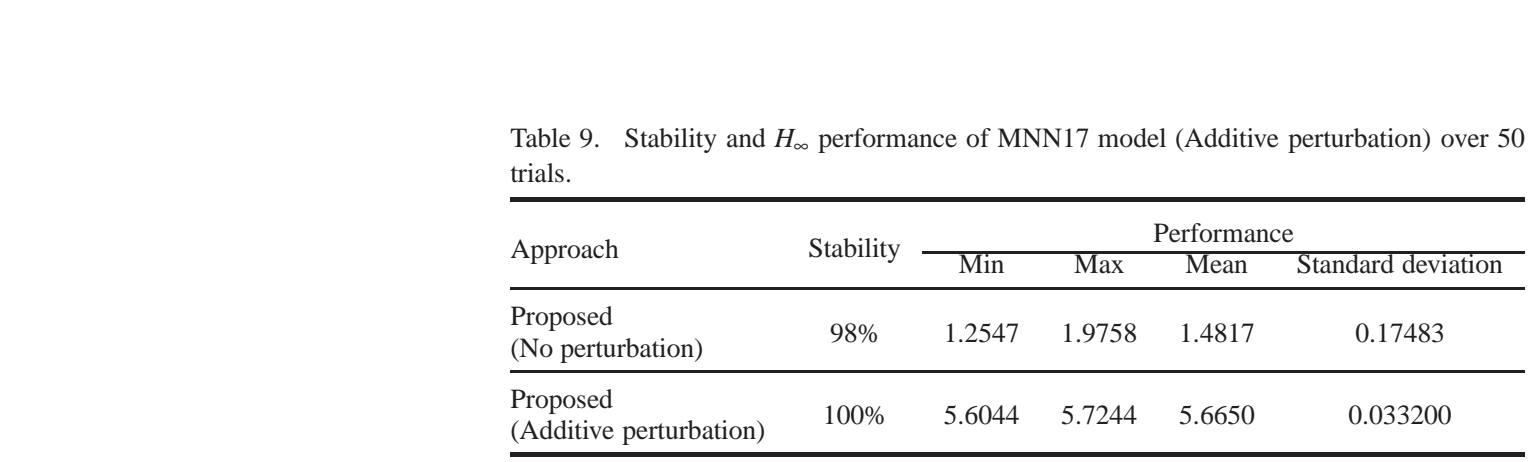

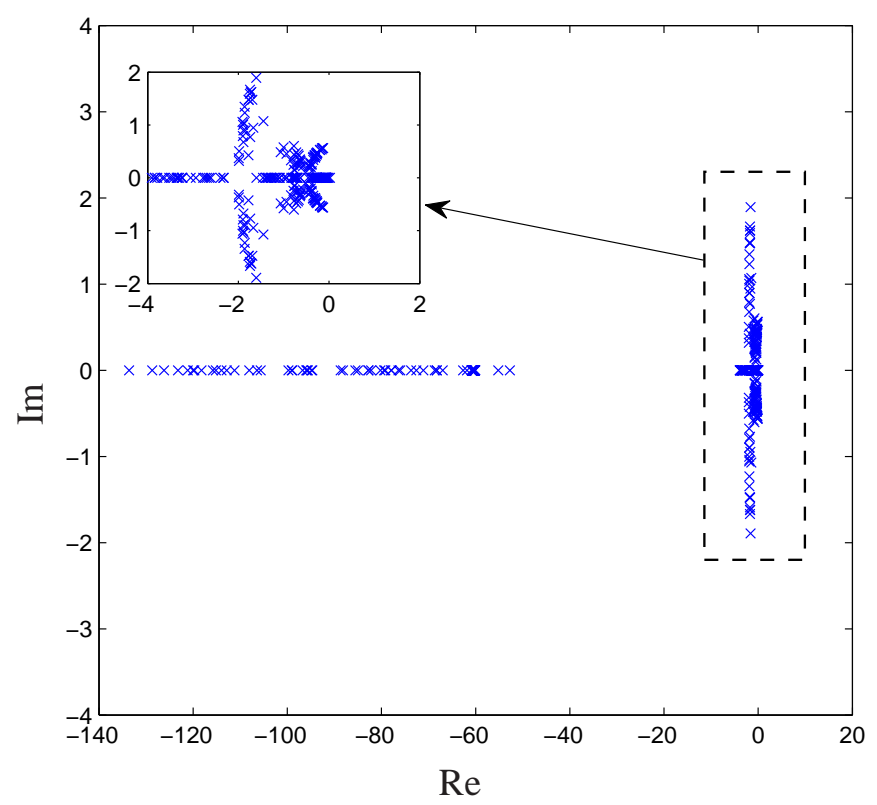

(a) Perturbed closed-loop poles with proposed nominal PID controller.

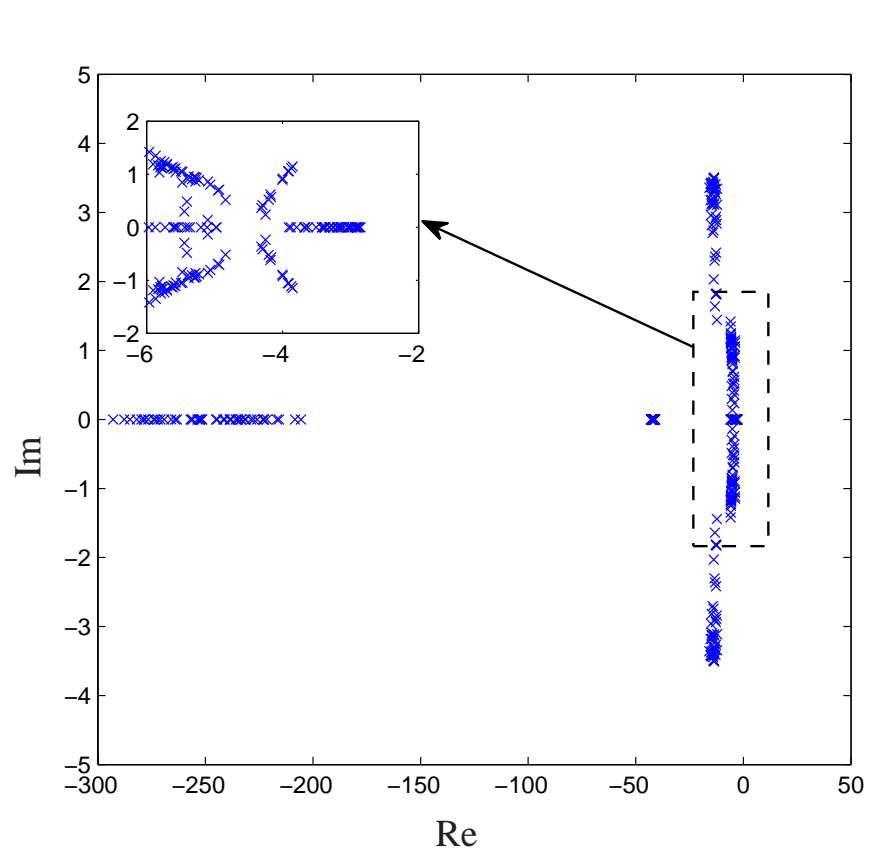

(b) Perturbed closed-loop poles with proposed non-fragile (additive) PID controller.

Figure 6. Perturbed closed-loop poles (MNN17) under additive perturbation. 
Table 10. Stability and $H_{\infty}$ performance of MNN17 model (Additive perturbation) over 50 trials.

\begin{tabular}{lccccc}
\hline \multirow{2}{*}{ Approach } & Stability & \multicolumn{4}{c}{ Performance } \\
\cline { 3 - 6 } & & Min & Max & Mean & Standard deviation \\
\hline $\begin{array}{l}\text { Proposed } \\
\text { (No perturbation) }\end{array}$ & $100 \%$ & 1.4185 & 1.4386 & 1.4285 & 0.0054154 \\
\hline $\begin{array}{l}\text { Proposed } \\
\text { (Multiplicative perturbation) }\end{array}$ & $100 \%$ & 5.3713 & 5.6382 & 5.5099 & 0.083601 \\
\hline
\end{tabular}

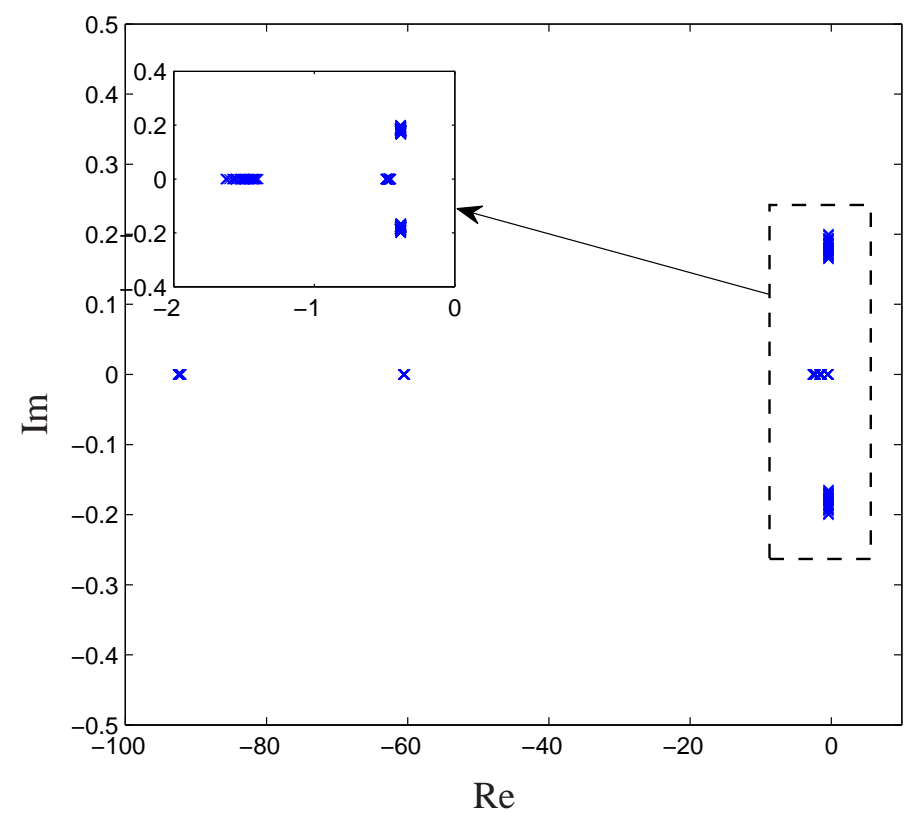

(a) Perturbed closed-loop poles with proposed nominal PID controller.

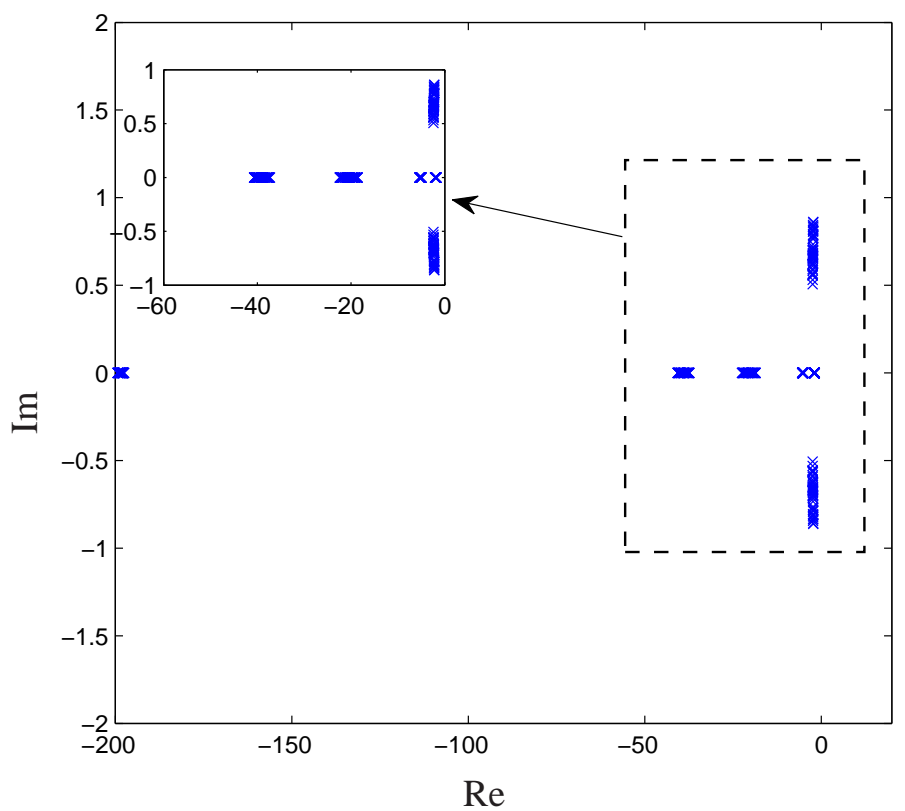

(b) Perturbed closed-loop poles with proposed non-fragile (multiplicative) PID controller.

Figure 7. Perturbed closed-loop poles (MNN17) under multiplicative perturbation.

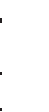

Multiplicative perturbation) 
rejection performance.

\section{Conclusions}

This paper has provided an LMI-based iterative approach for non-fragile multivariable PID controller design. The main idea of proposed approaches is to transform the PID controller design problem into an SOF control problem by extending the original system to an equivalent continuous-time system. Additive gain perturbations and multiplicative gain perturbations have been considered in the PID controller design problem. By virtue of the system augmentation approach, the conditions with slack matrices for solving the multivariable PID controller gains have been established and LMI based iterative algorithms have been provided to solve the conditions. Simulation results and comparison with other approaches have demonstrated the effectiveness and advantages of the proposed approach.

\section{References}

Åström, K.J., and Hägglund, T., Advanced PID Control, ISA-The Instrumentation, Systems, and Automation Society; Research Triangle Park, NC 27709 (2006).

Bettou, K., and Charef, A. (2009), "Control quality enhancement using fractional $\mathrm{PI}^{\lambda} \mathrm{D}^{\mu}$ controller," International Journal of Systems Science, 40(8), 875-888.

Bianchi, F.D., Mantz, R.J., and Christiansen, C.F. (2008), "Multivariable PID control with set-point weighting via BMI optimisation," Automatica, 44(2), 472-478.

Bolea, Y., Puig, V., and Blesa, J. (2014), "Gain-scheduled Smith Predictor PID-based LPV controller for open-flow canal control," IEEE Transactions on Control Systems Technology, 22(2), 468-477.

Che, W., Yang, G.H., and Jin, X. (2014), "Non-fragile $H_{\infty}$ filter design with sparse structure for linear discrete-time systems," Journal of the Franklin Institute, 351(1), 225-240.

De Oliveira, M.C., Geromel, J.C., and Hsu, L. (1999), "LMI characterization of structural and robust stability: the discrete-time case," Linear Algebra and Its Applications, 296(1), 27-38.

Du, H., Lam, J., and Sze, K.Y. (2003), "Non-fragile output feedback $H_{\infty}$ vehicle suspension control using genetic algorithm," Engineering Applications of Artificial Intelligence, 16(7), 667-680.

Fergani, N., and Charef, A. (2016), "Process step response based fractional $\mathrm{PI}^{\lambda} \mathrm{D}^{\mu}$ controller parameters tuning for desired closed loop response," International Journal of Systems Science, 47(3), 521-532.

Gahinet, P., and Apkarian, P. (1994), "A linear matrix inequality approach to $H_{\infty}$ control," International Journal of Robust and Nonlinear Control, 4(4), 421-448.

Geromel, J.C., and Colaneri, P. (2006), "Robust stability of time varying polytopic systems," Systems \& Control Letters, 55(1), 81-85.

Gil, P., Lucena, C., Cardoso, A., and Palma, L.B. (2015), "Gain Tuning of Fuzzy PID Controllers for MIMO Systems: A Performance-Driven Approach," IEEE Transactions on Fuzzy Systems, 23(4), 757-768.

Gündeş, A.N., Mete, A.N., and Palazoğlu, A. (2009), "Reliable decentralized PID controller synthesis for two-channel MIMO processes," Automatica, 45(2), 353-363.

Hägglund, T. (2012), "Signal filtering in PID control," in Proceedings of IFAC Conference on Advances in PID Control (PID'12).

Hägglund, T. (2013), "A unified discussion on signal filtering in PID control,” Control Engineering Practice, 21(8), 994-1006.

Halevi, Y., Palmor, Z.J., and Efrati, T. (1997), "Automatic tuning of decentralized PID controllers for MIMO processes," Journal of Process Control, 7(2), 119-128.

He, Y., and Wang, Q.G. (2006), "An Improved ILMI Method for Static Output Feedback Control With Application to Multivariable PID Control," IEEE Transactions on Automatic Control, 51(10), 1678-1683.

Ho, M.T. (2000), "Non-fragile PID controller design," in Proceedings of the 39th IEEE Conference on Decision and Control, 2000, Vol. 5, pp. 4903-4908.

Ho, M.T., Datta, A., and Bhattacharyya, S.P. (2001), "Robust and non-fragile PID controller design," International Journal of Robust and Nonlinear Control, 11(7), 681-708.

Hu, J., Liang, J., Chen, D., Ji, D., and Du, J. (2015), "A recursive approach to non-fragile filtering for networked 
systems with stochastic uncertainties and incomplete measurements," Journal of the Franklin Institute, 352(5), 1946-1962.

Huang, S., and Yang, G.H. (2015), "Non-fragile $H_{\infty}$ dynamic output feedback control for uncertain Takagi-Sugeno fuzzy systems with time-varying delay,” International Journal of Systems Science, 47(12), 2954-2964.

Jin, Q., and Liu, Q. (2014), "Multi-loop PI/PID controllers design for disturbance rejection based on non-parametric effective model and non-convex optimisation," IET Control Theory Applications, 8(15), 1499-1512.

Jung, J.W., Leu, V.Q., Do, T.D., Kim, E.K., and Choi, H.H. (2015), “Adaptive PID speed control design for permanent magnet synchronous motor drives," IEEE Transactions on Power Electronics, 30(2), 900-908.

Keel, L.H., and Bhattacharyya, S.P. (1997), "Robust, fragile, or optimal?," IEEE Transactions on Automatic Control, 42(8), 1098-1105.

Knospe, C. (2006), "PID control," IEEE Control Systems Magazine, 26(1), 30-31.

Leibfritz, F., "COMPl $l_{e} i b$ : COnstrained Matrix-optimization Problem library - a collection of test examples for nonlinear semidefinite programs, control system design and related problems," (2003).

Li, Y., Ang, K.H., and Chong, G.C.Y. (2006), "PID control system analysis and design,” IEEE Control Systems, 26(1), 32-41.

Li, Z., Wang, Z., Ding, D., and Shu, H. (2015), "Non-fragile $H_{\infty}$ control with randomly occurring gain variations, distributed delays and channel fadings," IET Control Theory \& Applications, 9(2), 222-231.

Lin, C., Wang, Q.G., and Lee, T.H. (2004), “An improvement on multivariable PID controller design via iterative LMI approach," Automatica, 40(3), 519-525.

Palmor, Z.J., Halevi, Y., and Krasney, N. (1995), "Automatic tuning of decentralized PID controllers for TITO processes," Automatica, 31(7), 1001-1010.

Romero Segovia, V., Hägglund, T., and Åström, K.J. (2013), "Noise filtering in PI and PID control," in Proceedings of American Control Conference, 2013, IEEE, pp. 1763-1770.

Shen, H., Wu, Z., Zhang, Z., and Park, J.H. (2014), "Non-fragile mixed $H_{\infty} / l_{2}-l_{\infty}$ synchronisation control for complex networks with Markov jumping-switching topology under unreliable communication links," IET Control Theory \& Applications, 8(18), 2207-2218.

Shu, Z., and Lam, J. (2009), "An augmented system approach to static output-feedback stabilization with performance for continuous-time plants," International Journal of Robust and Nonlinear Control, 19(7), 768-785.

Shu, Z., Lam, J., and Xiong, J. (2009), "Non-Fragile Exponential Stability Assignment of Discrete-Time Linear Systems With Missing Data in Actuators," IEEE Transactions on Automatic Control, 54(3), 625-630.

Tandon, A., and Dhawan, A. (2016), "Non-fragile robust optimal guaranteed cost control of uncertain 2-D discrete state-delayed systems," International Journal of Systems Science, 47(14), 1464-5319.

Vu, T.N.L., and Lee, M. (2010), "Independent design of multi-loop PI/PID controllers for interacting multivariable processes," Journal of Process control, 20(8), 922-933.

Wang, Q.G., Zou, B., Lee, T.H., and Bi, Q. (1997), “Auto-tuning of multivariable PID controllers from decentralized relay feedback," Automatica, 33(3), 319-330.

Wu, M., Yan, J., She, J.H., and Cao, W.H. (2009), "Intelligent decoupling control of gas collection process of multiple asymmetric coke ovens," IEEE Transactions on Industrial Electronics, 56(7), 2782-2792.

Wu, Z.Z., Iqbal, A., and Amara, F.B. (2011), "LMI-based multivariable PID controller design and its application to the control of the surface shape of magnetic fluid deformable mirrors," IEEE Transactions on Control Systems Technology, 19(4), 717-729.

Xie, L., de Souza, C.E., and Fragoso, M.D. (1991), " $H_{\infty}$ filtering for linear periodic systems with parameter uncertainty," Systems \& Control Letters, 17(5), 343-350.

Xu, S., Lam, J., Wang, J., and Yang, G.H. (2004), "Non-fragile positive real control for uncertain linear neutral delay systems," Systems \& Control Letters, 52(1), 59-74.

Yang, G.H., and Che, W. (2008), "Non-fragile $H_{\infty}$ filter design for linear continuous-time systems," Automatica, 44(11), 2849-2856.

Yang, G.H., and Wang, J. (2001), "Non-fragile $H_{\infty}$ control for linear systems with multiplicative controller gain variations," Automatica, 37(5), 727-737.

Yu, W., and Rosen, J. (2013), "Neural PID control of robot manipulators with application to an upper limb exoskeleton," IEEE Transactions on Cybernetics, 43(2), 673-684.

Yue, D., and Lam, J. (2005), "Non-fragile guaranteed cost control for uncertain descriptor systems with time-varying state and input delays," Optimal Control Applications and Methods, 26(2), 85-105.

Zhang, D., Cai, W., and Wang, Q.G. (2014), "Robust non-fragile filtering for networked systems with distributed variable delays," Journal of the Franklin Institute, 351(7), 4009-4022.

Zhang, H., Shi, Y., and Mehr, A.S. (2012), "Robust $H_{\infty}$ PID control for multivariable networked control systems with 
disturbance/noise attenuation," International Journal of Robust and Nonlinear Control, 22(2), 183-204.

Zhang, Y., Shieh, L.S., and Dunn, A.C. (2004), "PID controller design for disturbed multivariable systems," IEE Proceedings-Control Theory and Applications, 151(5), 567-576.

Zheng, F., Wang, Q.G., and Lee, T.H. (2002), “On the design of multivariable PID controllers via LMI approach,"

Automatica, 38(3), 517-526. 